\title{
Pickup ion-mediated plasma physics of the outer heliosphere and very local interstellar medium
}

\author{
G. P. Zank
}

\begin{abstract}
Observations of plasma and turbulence in the outer heliosphere (the distant supersonic solar wind and the subsonic solar wind beyond the heliospheric termination shock) made by the Voyager Interstellar Mission and the energetic neutral atom observations made by the IBEX spacecraft have revealed that the underlying plasma in the outer heliosphere and very local interstellar medium (VLISM) comprises distinct thermal proton and electron and suprathermal pickup ion (PUI) populations. Estimates of the appropriate collisional frequencies show that the multi-component plasma is not collisionally equilibrated in either the outer heliosphere or VLISM. Furthermore, suprathermal PUIs in these regions form a thermodynamically dominant component. We review briefly a subset of the observations that led to the realization that the solar wind-VLISM interaction region is described by a non-equilibrated multi-component plasma and summarizes the derivation of suitable plasma models that describe a PUI-mediated plasma.
\end{abstract}

Keywords: Solar wind, Interstellar medium, Plasma, Pickup ions, Neutral gas

\section{Introduction}

The Voyager 1 (V1) spacecraft crossed the heliopause, the boundary separating matter of solar origin from interstellar matter, and entered the local interstellar medium (LISM) during August 2012 (Stone et al. 2013; Krimigis et al. 2013; Burlaga et al. 2013; Gurnett et al. 2013), an event of enormous historical import for humankind. Voyager 1 is the first human-made object to leave the confines of the heliosphere and enter interstellar space. With a working set of instruments, Voyager 1 begins an epoch of extraordinary in situ discovery science in the interstellar medium. We now have the opportunity to study in situ basic plasma physical processes in the interstellar medium (ISM). We review briefly our understanding of the basic plasma physics model that is beginning to emerge as a result of observations made by the Voyager interstellar mission (Voyagers 1 and 2) and the

*Correspondence: garyp.zank@gmail.com

Center of Space Plasma and Aeronomic Research and Department of Space Science, University of Alabama in Huntsville, Sparkman Ave, Huntsville, AL 35899, USA interstellar boundary explorer (IBEX) of and in our very local neighborhood of the LISM.

It is now recognized that the interstellar medium and heliosphere are coupled intimately through charge exchange of neutral $\mathrm{H}$ and protons, and that the physics of the outer heliosphere and neighboring LISM cannot be understood independently of each other.

The heliosphere is the region of space filled by the expanding solar corona; a region extending $>120$ astronomical units (AU) in the direction of the Sun's motion through the interstellar medium and perhaps tens of thousands of $\mathrm{AU}$ in the opposite or heliotail direction. Neutral interstellar hydrogen is the dominant (by mass) constituent of the solar wind beyond an ionization cavity of $\sim 6-10 \mathrm{AU}$ in the upwind direction (the direction antiparallel to the incident interstellar wind), and is coupled weakly to the solar wind plasma via resonant charge exchange. Charge exchange produces pickup ions (PUIs) that eventually dominate the internal energy of the solar wind.

If, for simplicity, we adopt initially a perspective that the plasma can be described as a single-fluid or magnetohydrodynamic (MHD) system, then the heliospheric-LISM 
plasma environment is composed of essentially three thermodynamically distinct regions: (i) the supersonic solar wind, with a relatively low temperature, large radial speeds, and low densities, bounded by the heliospheric termination shock (HTS). The outer heliosphere is that region of the solar wind influenced dynamically by physical processes associated with the LISM. (ii) The transition of the supersonic solar wind to a subsonic flow through the HTS creates a region of heated subsonic solar wind, called the inner heliosheath (IHS). The IHS has much higher temperatures and densities, larger magnetic fields, and lower flow speeds than does the distant supersonic solar wind (SW). The IHS is bounded by a contact or tangential discontinuity called the heliopause (HP). (iii) The HP is the boundary that separates plasma of solar origin from plasma of interstellar origin. The LISM in our neighborhood possesses a small plasma flow speed and temperature, but the density is higher than in regions (i) and (ii). A bow shock may or may not exist ahead of the heliosphere due to the relative motion of the Sun and interstellar medium. The three regions are illustrated in Fig. 1 (left panel), where region 3 corresponds to the supersonic solar wind, region 2 to the hot inner heliosheath, and the interstellar region is subdivided into region $1 \mathrm{~b}$ between the HP and a possible bow shock/ wave, sometimes called the outer heliosheath (OHS), and region 1a beyond a bow shock or bow wave. Of course, as we discuss below, the plasma system is vastly more complicated than that of MHD and the plasma itself possesses multiple components coupled via charge-exchange and/or collisional and collisionless processes, with associated transfer of charge, momentum, and energy, and thus thermodynamic coupling. Nonetheless, the zerothorder distinction of thermodynamically distinct regions provides a useful intuitive guide to the underlying physics of the global solar wind-LISM interaction.

Each of the thermodynamically distinct regions is the source of a distinct population of hydrogen $(\mathrm{H})$ atoms produced by charge exchange between the ambient plasma and neutrals entering the region (Zank et al. 1996). These three distinct neutral $\mathrm{H}$ populations include the "splash" component produced in the fast or supersonic solar wind, i.e., fast neutrals that acquire high radially outward speeds $(\sim 400-750 \mathrm{~km} / \mathrm{s})$ with a relatively small thermal spread, very hot neutrals produced in the inner heliosheath with comparatively high speeds $(\sim 100 \mathrm{~km} / \mathrm{s})$ and a large thermal spread (which can produce ENAs with speeds even that exceed $100 \mathrm{~km} / \mathrm{s}$ ), and decelerated heated atoms originating in the outer heliosheath.

The charge-exchange mean free path (mfp) of neutral hydrogen atoms in the LISM (region 1) is approximately $\sim 100$ AU (assuming a charge-exchange cross-section $\sigma_{c}=5 \times 10^{-15} \mathrm{~cm}^{2}$ and a total LISM number density of $0.2 \mathrm{~cm}^{-3}$ ), in the IHS (region 2) $\sim 2500 \mathrm{AU}$ for a number density of $0.005 \mathrm{~cm}^{-3}$, and $>200 \mathrm{AU}$ in the supersonic solar wind beyond 10 AU (region 3). With the exception of the local interstellar medium region, the chargeexchange mfps are so large that they exceed the expected scale size of the boundary regions separating the heliosphere and LISM. The interaction of the solar wind with the LISM therefore requires the modeling of plasmas and non-equilibrated $\mathrm{H}$ atom gas. Despite the very large charge-exchange mfps in both the supersonic solar wind and the boundary regions, the structure of the global heliosphere is determined in large part by the non-equilibrated coupling of neutral interstellar $\mathrm{H}$ to supersonic and subsonic solar wind plasma (Zank 1999; 2015; Zank et al. 2009; McComas et al. 2011). This makes the modeling of the solar wind interaction with the LISM very challenging. Nonetheless, despite these complications, the basic structure illustrated in the cartoon Fig. 1 (left) emerges from simulations that include the basic physics of the plasma-H charge-exchange coupling. An illustrative simulation of a 2D coupled model of the heliospheric interaction with the LISM is shown in Fig. 1 (right panel). The top plot shows the 2D plasma temperature distribution, clearly identifying the three distinct regions and the overall topology and boundaries that can exist (together with a further sub-division of region 1 into pre- and postbow shock regions $1 \mathrm{a}$ and $1 \mathrm{~b}$, respectively). The bottom plot illustrates the neutral $\mathrm{H}$ density distribution. A more extended summary that discusses the magnetic field observations in both the IHS and at the HP, together with associated references and related theoretical modeling, can be found in the review by Zank (2015).

The coupling of plasma and neutral $\mathrm{H}$ occurs through the creation of PUIs via charge exchange between the charged and neutral gases. Over suitably large distances, the neutral $\mathrm{H}$ and protons are fully equilibrated, both possessing the same temperature and velocity. Charge exchange in a fully equilibrated partially ionized plasma has no essential dynamical effect, with charge exchange effectively doing no more than relabeling protons and $\mathrm{H}$ atoms (assuming that the dominant neutral gas component is $\mathrm{H}$ atoms-in the LISM, this is a reasonable assumption, although $\mathrm{He}$ atoms are approximately $9 \%$ of the neutral gas and the remaining heavy atom neutral gas is about $1 \%$ ). However, in regions 2 and 3, the interstellar $\mathrm{H}$ drift speed is different from the plasma flow velocity $(\sim 20 \mathrm{~km} / \mathrm{s}$ for $\mathrm{H}$ versus $\sim 100-750 \mathrm{~km} / \mathrm{s}$ for the plasma), and $\mathrm{H}$ originating from regions 3 and 2 that splashes back into the LISM has flow speeds ranging from $\sim 100->400 \mathrm{~km} / \mathrm{s}$, which is quite different from the $\sim 15-26 \mathrm{~km} / \mathrm{s}$ speed of region 1 . Thus, throughout the boundary regions and in the LISM within several 100 


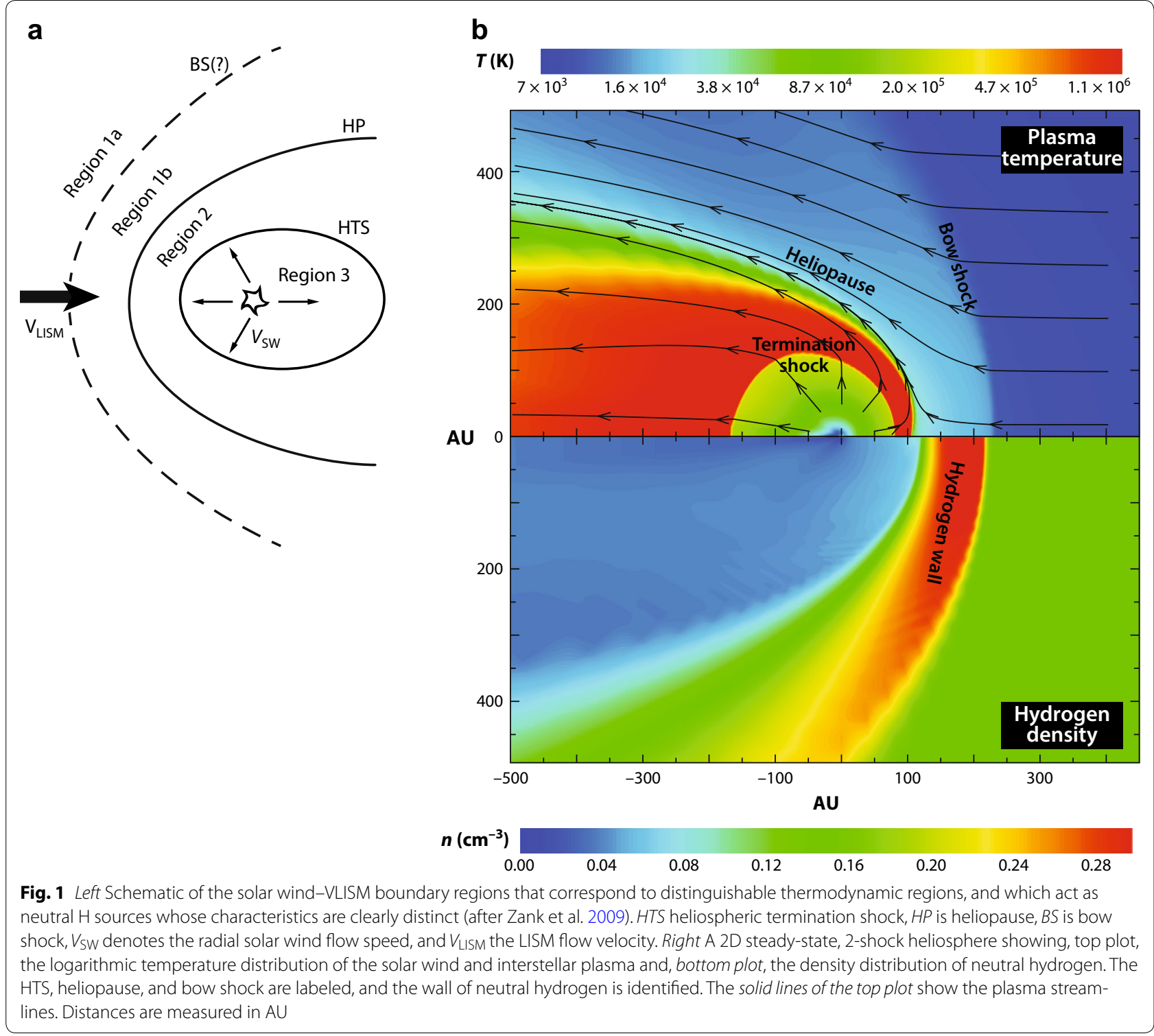

AU of the HP, there is a relative drift between the background plasma and some $\mathrm{H}$ components. Depending on the specific environment, the neutral gas can be ionized by either solar photons (photoionization) or charged particles (charge exchange, electron-impact ionization) and the new ions are accelerated almost instantaneously by the motional electric field of the plasma. The PUIs form a ring-beam distribution on the time scale of the inverse gyrofrequency and stream along the magnetic field while experiencing advection by the bulk plasma flow perpendicular to the mean magnetic field. Newly created PUIs drive a host of plasma instabilities, from fast magnetosonic and Alfvénic waves, ion cyclotron waves, to lower hybrid waves (e.g., Lee and Ip 1987; Cairns and Zank
2002; Gary and Madland 1988, see Gary 1991; Isenberg 1995; Zank 1999 for extensive summaries). PUIs experience scattering and gradual isotropization by either ambient or self-generated low-frequency electromagnetic fluctuations in the plasma. Since the newly born ions are eventually isotropized, their bulk velocity is essentially that of the background plasma, i.e., they advect with the plasma flow and are then said to be "picked up" by the flowing plasma. The isotropized PUIs form a distinct suprathermal population of energetic ions $(\sim 1 \mathrm{keV}$ energies in the supersonic $S W$, with a number density approximately $20 \%$ of the solar wind number density in the vicinity of the HTS) in the plasma whose origin is either the interstellar medium when considering region 3 and 2 
or the heliosphere when considering regions 2 and 1 (e.g., Holzer 1972; Lee and Ip 1987; Williams and Zank 1994, see Zank 1999, 2015 for an extensive review).

Consider now the three specific regions discussed above. PUIs are created in these regions and mediate the plasma properties. Although each region is mediated by PUIs, the origin of the PUI population in each is different in important ways.

Coulomb collisions are necessary to thermally equilibrate a background thermal plasma, such as the solar wind, and the PUI protons. In the case of the supersonic solar wind, (Isenberg 1986) argued that a multi-fluid model is necessary to describe a coupled solar windPUI plasma since neither proton nor electron collisions can equilibrate the PUI-mediated supersonic solar wind plasma (see Zank et al. 2014).

The inner heliosheath (IHS) is complicated by the microphysics of the HTS. The supersonic solar wind is decelerated on crossing the quasi-perpendicular HTS. The flow velocity is directed away from the radial direction and is $\sim 100 \mathrm{~km} / \mathrm{s}$. The interplanetary magnetic field remains approximately perpendicular to the plasma flow. Voyager 2 measured the downstream solar wind temperature to be in the range of $\sim 120,000-180,000 \mathrm{~K} \sim 16$ eV (Richardson 2008; Richardson et al. 2008), which was much less than predicted by simple MHD models. Instead, the thermal energy in the IHS is dominated by PUIs. There are two primary sources of PUIs in the inner heliosheath. The first is interstellar neutrals that drift across the HP and charge exchange with hot solar wind plasma. These newly created ions are picked up in the IHS plasma in the same way that ions are picked up in the supersonic solar wind. The characteristic energy for PUIs created in this manner is $\sim 50 \mathrm{eV}$ or $\sim 6 \times 10^{5} \mathrm{~K}$, which is about five times hotter than the IHS solar wind protons. The second primary source is PUIs created in the supersonic solar wind and then convected across the HTS into the IHS. The PUIs convected to the HTS are either transmitted immediately across the HTS or are reflected before transmission (Zank et al. 1996). PUI reflection was predicted by Zank et al. (1996) to be the primary dissipation mechanism at the quasi-perpendicular HTS, with the thermal solar wind protons experiencing comparatively little heating across the HTS. The transmitted PUIs downstream of the HTS have temperatures $\sim 9.75 \times 10^{6} \mathrm{~K}(\sim 0.84 \mathrm{keV})$ and the reflected protons have a temperature of $\sim 7.7 \times 10^{7} \mathrm{~K}(\sim 6.6 \mathrm{keV})$ (Zank et al. 2010). PUIs, whether transmitted, reflected, or injected, dominate the thermal energy of the IHS, despite being only some $20 \%$ of the thermal subsonic solar wind number density at the HTS. The IHS proton distribution function can be approximated by a 3(Zank et al. 2010; Burrows et al. 2010) or 4-component distribution function (Zirnstein et al. 2014), with a relatively cool thermal solar wind Maxwellian distribution and two or three superimposed PUI distributions. Such a decomposition of the IHS proton distribution function can be exploited in modeling energetic neutral atom (ENA) spectra observed by the IBEX spacecraft at $1 \mathrm{AU}$ (Desai et al. 2012; Zirnstein et al. 2014; Desai et al. 2014). Multiple proton populations were identified in the IHS and the very local interstellar medium, these being the various PUI populations described above and the thermal solar wind proton population (Zank et al. 2010). Zank et al. (2014) show that in the IHS neither proton nor electron collisions can equilibrate a PUI-thermal solar wind plasma in the subsonic solar wind or IHS on scales smaller than at least $10,000 \mathrm{AU}$, meaning that a multi-component plasma description that discriminates between PUIs and the subsonic solar wind plasma is necessary.

The interstellar plasma upwind of the heliopause is also mediated by energetic PUIs. It was noted (Zank et al. 1996) that energetic neutral $\mathrm{H}$ created via charge exchange in the IHS and fast solar wind could "splash" back into the interstellar medium where they would experience a secondary charge exchange. The secondary charge exchange of hot and/or fast neutral $\mathrm{H}$ with cold ( 7500 K-McComas et al. (2012, 2015); Schwadron et al. (2015); Bzowski et al. (2015) LISM protons leads to the creation of a hot or suprathermal PUI population locally in region 1 . The heating of the LISM in the neighborhood of the Sun has been discussed in detail (Zank et al. 2013), since this results in an increased sound speed with a concomitant weakening or even elimination of the bow shock (yielding instead a bow wave) (McComas et al. 2012). PUIs form a tenuous $\left(n_{p} \simeq 5 \times 10^{-5} \mathrm{~cm}^{-3}\right.$, (Zirnstein et al. 2014) suprathermal component in the plasma upwind of the HP that is not collisionally equilibrated in the LISM on scales smaller than at least $75 \mathrm{AU}$ (Zank et al. 2014).

Zank (2015) introduced an alternative definition of the very local interstellar medium (VLISM) than has been used previously (Holzer 1989). Instead of defining the VLISM as the region within $0.01 \mathrm{pc}$ of the Sun, i.e., within about $2000 \mathrm{AU}$ (Holzer 1989), the definition that we advocate is that it is that region of the ISM surrounding the Sun which is modified by the deposition of heliospheric material. A corresponding definition would be that the VLISM is a part of the ISM surrounding the Sun that is not equilibrated with either $\mathrm{H}$ or PUIs of heliospheric origin. This is consistent with the discussion above, and simulations (Zank et al. 2013; Heerikhuisen et al. 2014) show that the interstellar temperature is modified by the creation of heliospheric PUIs to at least some $700 \mathrm{AU}$ of the Sun in the upwind direction. 


\section{Review}

\section{Selected observations}

The crossing of the HTS by Voyager 2 (V2) revealed an almost classical perpendicular shock structure (labeled TS-3) (Burlaga et al. 2008; Richardson et al. 2008), except that the observed average downstream proton plasma temperature was an order of magnitude smaller than predicted by the MHD Rankine-Hugoniot conditions (Zank et al. 2009). The transmitted solar wind proton distribution is a broadened/heated Maxwellian (with a somewhat flattened peak), and there is no evidence of reflected solar wind ions being transmitted downstream (Richardson et al. 2008; Richardson 2008). Richardson et al. (2008); Richardson (2009) concluded that PUIs provide both the primary shock dissipation mechanism and the bulk of the hot plasma downstream of the HTS, as predicted 12 years earlier by Zank et al. (1996). The basic model (Zank et al. 1996) for the microstructure of the HTS therefore appears to be supported by V2 observations. However, both the observed solar wind proton distribution and a shock dissipation mechanism based on PUIs mean that the downstream proton distribution function is a (possibly complicated) function of the physics of the HTS. Zank et al. (2010) developed a basic model of a quasi-perpendicular HTS, mediated by PUIs, to derive the complete downstream proton distribution function in the IHS, determine the partitioning of energy between solar wind protons and PUIs, and infer the implications of the constructed IHS proton distribution function for the ENA spectral flux observed by IBEX.

Zank et al. (2010) introduced a three-distribution approximation of the IHS proton distribution, comprising core solar wind protons, transmitted (without reflection) PUIs, and reflected (and then transmitted) PUIs. Electrons are of course included too in the complete plasma model. The reflected PUI population results from the reflection of some upstream PUIs at the cross-shock electrostatic potential of the quasi-perpendicular HTS. Reflected PUIs are the primary dissipation mechanism at the HTS (Zank et al. 1996; Lipatov and Zank 1999; Burrows et al. 2010). Although the post-HTS PUI distribution is likely highly complex, as a first approximation the solar wind proton distribution is a Maxwellian. Since the number of PUIs reflected is comparatively small, a simplifying assumption that the non-reflected PUI distribution can be approximated by either a filled-shell or a Maxwellian distribution can be made (Zank et al. 2010). The downstream PUI temperatures for the transmitted and reflected PUIs can be computed (Zank et al. 2010), allowing the partitioning of downstream thermal energy into transmitted solar wind protons, transmitted PUIs and reflected, and then transmitted PUIs to be determined. The smoothed form of the constructed heliosheath proton distribution (Zank et al. 2010) resembles a $\kappa$-distribution (Heerikhuisen et al. 2008). As a result, a significant number of protons reside in the wings of the distribution function, quite unlike the Maxwellian distribution. The close correspondence between the constructed distributions and the $\kappa$-distribution with index 1.63 is useful in allowing for simplified simulations based on a $\kappa$-distribution (Heerikhuisen et al. 2008; Zank et al. 2010, 2013; Zank 2015). Zank et al. (2010) predicted that the constructed heliosheath proton distribution should possess some structure that would manifest itself in ENA spectra observed at 1 AU by IBEX, and that the microphysics of the HTS plays a key role in determining the form of the total downstream or heliosheath proton distribution. Finally, we note that kinetic hybrid and PIC simulations (Lipatov and Zank 1999; Oka et al. 2011; Wu et al. 2009; Matsukiyo and Scholer (2011, (2014); Yang et al. 2015) appear to support the basic model (Zank et al. 1996, 2010) rather well. These comments are illustrated graphically in Fig. 2, where we show three panels. The left panel plots the solar wind proton distribution upstream and downstream of the HTS observed by the Voyager 2 plasma instrument PLS (Richardson 2008). Unfortunately, the PLS instrument measures only solar wind energy protons and not PUIs. The observed downstream proton distribution shows clearly that the transmitted solar wind distribution is simply a heated Maxwellian distribution and no reflected solar wind protons can be identified. The middle panel shows the theoretically predicted total downstream proton velocity distribution function Zank et al. (2010). The blue curve shows a $\kappa$-distribution with a kappa value of 1.63 (Heerikhuisen et al. 2008). The black curves depict the distribution constructed from a superposition of transmitted solar wind protons, transmitted but not reflected PUIs, and reflected and then transmitted PUIs. The red curve illustrates a Maxwellian distribution for the observed downstream density and temperature. For this model, the heliosheath constructed proton distribution (black curve) assumed that downstream phase mixing of PUIs caused them to evolve into an approximately Maxwellian distribution. The assumption of a downstream PUI distribution intermediate to a filled shell and a Maxwellian distribution creates a shoulder feature in the total downstream proton distribution function (Zank et al. 2010). The right panel shows the total transmitted solar wind and PUI distribution function downstream of the HTS derived from a hybrid simulation (Oka et al. 2011) - see also Yang et al. (2015) - assuming an upstream PUI number density of $30 \%$ of the total proton number density. The solar wind protons and the PUIs are denoted by the solid and dashed curves, respectively, and the heavy black curves are their sum. Besides illustrating the correspondence of the basic 

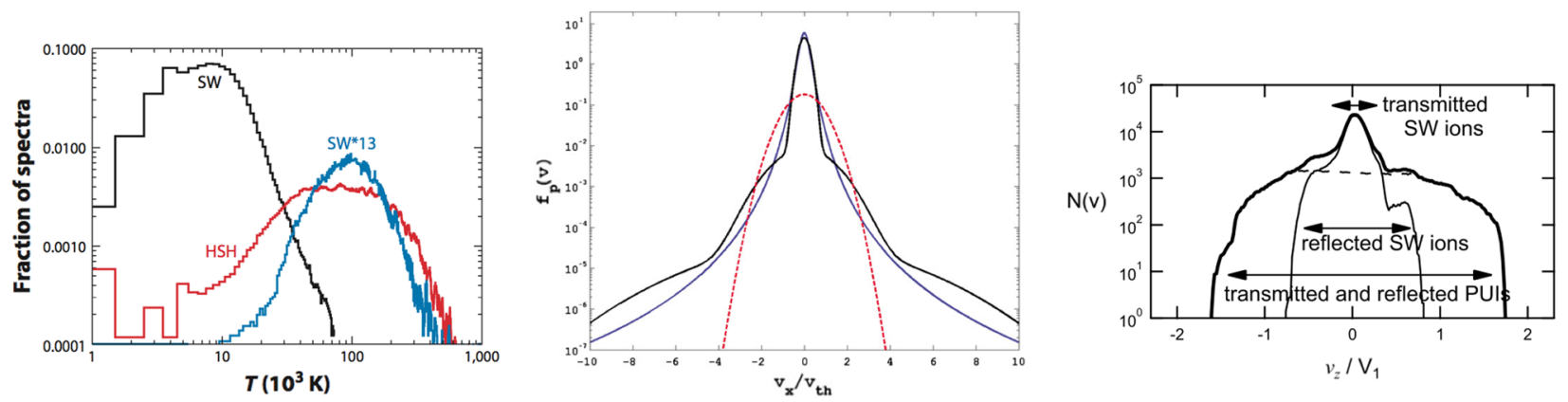

Fig. 2 Left histograms of the solar wind thermal proton temperature distributions observed by Voyager 2 across the HTS measured in the SW and IHS: (black) solar wind distribution, (red) IHS distribution, and (blue) distribution of the solar wind temperature multiplied by 13 , the ratio between the upstream solar wind and downstream IHS temperatures. No reflected solar wind protons can be identified from the distribution function (Richardson 2008). Center the IHS constructed proton distribution (black curve) assuming that the transmitted but not reflected PUls evolve into a Maxwellian distribution. The blue curve shows a $\kappa$-distribution with a value of -1.63 . The black curve is the superposition of transmitted solar wind protons, transmitted PUls, and reflected and then transmitted PUIs. The red curve is a Maxwellian distribution assuming the observed downstream density and temperature. The particle velocity $v_{x}$ is normalized to the Maxwellian thermal speed $v_{t h}=\sqrt{2 k T / m_{p}}$, where $k$ is Boltzmann's constant, $m_{p}$ the proton mass, and $T$ the total downstream temperature (Zank et al. 2010). Right one-dimensional cut of the proton velocity distribution function downstream of the HTS. The particle velocities are normalized by the upstream flow speed $V_{1}$ in the shock rest frame. The solar wind protons and the PUls are identified by the solid and dashed curves, respectively, and the thick black curves are their sum (Oka et al. 2011)

theory introduced in Zank et al. (2010) to both Voyager 2 observations and simulations, the relative energies of downstream solar wind ions and transmitted (both directly and initially reflected) PUIs are clearly illustrated in Fig. 2.

To test the possibility that the microphysics of the HTS would manifest itself in IBEX ENA spectra observed at 1 AU, Desai et al. (2012) in an initial study found that the fluxes, energy spectra, and energy dependence of the spectral indices of $\sim 0.5-6 \mathrm{keV}$ ENAs measured by IBEX-Hi along the V1 and V2 lines of sight were consistent within a factor of $\sim 2$ with the model results of Zank et al. (2010). The observed ENA spectra do not exhibit sharp cutoffs at approximately twice the solar wind speed as is typically found for shell-like PUI distributions in the heliosphere. Desai et al. concluded that the ENAs measured by IBEX-Hi are generated by at least two types of PUI populations whose relative contributions depend on the ENA energy: transmitted PUIs in the $\sim 0.5$ to $5 \mathrm{keV}$ energy range and reflected PUIs above $\sim 5 \mathrm{keV}$ energy (Desai et al. 2012).

The absence of sharp cutoffs in the ENA distribution indicates that the ENA source in the $\sim 0.5-5 \mathrm{keV}$ energy range is almost certainly beyond the HTS. The PUI distribution is modified by energization processes in the supersonic solar wind, such as the formation of the filled shell due to cooling, and it appears to develop an extended tail beyond $v-2 U$ ( $U$ the bulk solar wind speed). However, the tail does not emerge smoothly from the flat-topped PUI distribution function but instead appears as a discontinuous, lower intensity attachment to the cutoff step at $v \sim 2 U$ of the filled shell distribution (see e.g., Gloeckler et al. 1994, 2001), and so a cutoff is still present. However, Gloeckler et al. $(1994,2001)$ show examples of the transmission of the solar wind PUI distribution through a CIR reverse shock. The flat-topped PUI distribution is transformed into a kappa-like distribution on transmission through the interplanetary shock, i.e., a Maxwellian-like core with an extended tail that emerges smoothly from the thermal distribution. These observations, together with the theory described above, suggest that the observed ENAs are generated primarily downstream of the HTS, after the PUI distribution has been processed by the HTS, rather than upstream in the supersonic solar wind. A further interesting point concerns PUI, and hence ENA, energies higher than $\sim 6 \mathrm{keV}$. Since all upstream PUIs are processed by the HTS, this produces PUIs in the $\sim 0.5-6 \mathrm{keV}$ energy range downstream which do not have a flat-topped distribution. These energetic PUIs make up some $20 \%$ of the proton number density. Additional energization may result either at the shock (via, e.g., diffusive shock acceleration (Senanayake et al. 2015) or further downstream (Zank et al. 2015), or deep in the IHS itself (Lazarian and Opher 2009; Fisk and Gloeckler 2009), and this would then lead to a modification of the total proton spectrum in the IHS. Although it is difficult to quantify the effect of additional proton energization in the IHS, if it produces a power law distribution from the energetic tail of the HTS-processed distribution, then this will simply ensure that (i) there is no cutoff at $\sim 6 \mathrm{keV}$; (ii) the intensity in the energy range $\sim 0.5-6 \mathrm{keV}$ will be reduced a little (bearing in mind that 
the new distribution is a power law), and (iii) this process is likely to be of more importance to higher energy ENA observations (such as the Cassini INCA observations of ENAs (Krimigis et al. 2009).

The observed lower energy ENAs (below $\sim 0.5 \mathrm{keV}$ ) are not well described by the theory (Zank et al. 2010), and most existing models underestimate the ENA fluxes between $\sim 0.05-0.5 \mathrm{keV}$ by an order of magnitude or more (Fuselier et al. 2012). To address the lower energies, Zirnstein et al. (2014) extended the Zank et al. (2010) model in two ways. First, they accounted for the extinction of solar wind protons and transmitted and reflected PUIs by charge exchange with interstellar neutral $\mathrm{H}$ in the composite proton distribution. The extinction process alters the distribution of energy in the IHS, compared to assuming that the relative energy densities of the core SW protons and the transmitted and reflected PUIs remain constant. Determining an accurate partitioning of the energy is essential for understanding the role that PUIs play in the heliosphere and its effect on H ENA flux.

The second extension introduced by Zirnstein et al. (2014) was to include ENAs from the VLISM that were created by PUIs. Although ENAs are created everywhere in the solar wind-LISM interaction region, ENAs produced in the IHS easily propagate into the VLISM before charge exchange occurs, creating a population of PUIs there. ENAs produced in the VLISM, however, do not easily charge exchange in the IHS, and therefore permeate the inner heliosphere and can be detected at $1 \mathrm{AU}$. One can similarly partition the VLISM energy into various proton populations (Zirnstein et al. 2014). The VLISM plasma consists mostly of protons, initially $\sim 7500 \mathrm{~K}$ in the pristine LISM (McComas et al. 2015; Schwadron et al. 2015; Bzowski et al. 2015), that are partially heated by charge exchange near the $\mathrm{H}$ wall and by crossing a bow wave (McComas et al. 2012; Zank et al. 2013). However, the increase in thermal energy of the VLISM plasma near the HP is also due to energetic PUIs, which are created from charge exchange between LISM protons and ENAs from the IHS (Zank et al. 1996). The majority of PUIs are in close proximity to the HP and drop off exponentially at larger distances due to the mean free path of their parent ENAs, and due to advection with the LISM flow toward the HP (Zirnstein et al. 2014). As with the IHS, Zirnstein et al. (2014) determine the VLISM PUI properties by partitioning the total energy from the plasma-neutral results between LISM protons and PUIs. Since ENAs from IHS protons may propagate into the VLISM and charge exchange to become PUIs, they treat the VLISM plasma as a five-component distribution, including protons from the core (and compressed) VLISM plasma, and PUIs created by charge exchange from IHS ENAs.

Figure 3a shows various sources of the $\mathrm{H}$ spectrum in the V1 and V2 direction based on an extended model (Zirnstein et al. 2014) with a comparison to the corrected IBEX data (Desai et al. 2014). The results illustrated in Fig. 3 are based on a single set of parameters that were introduced
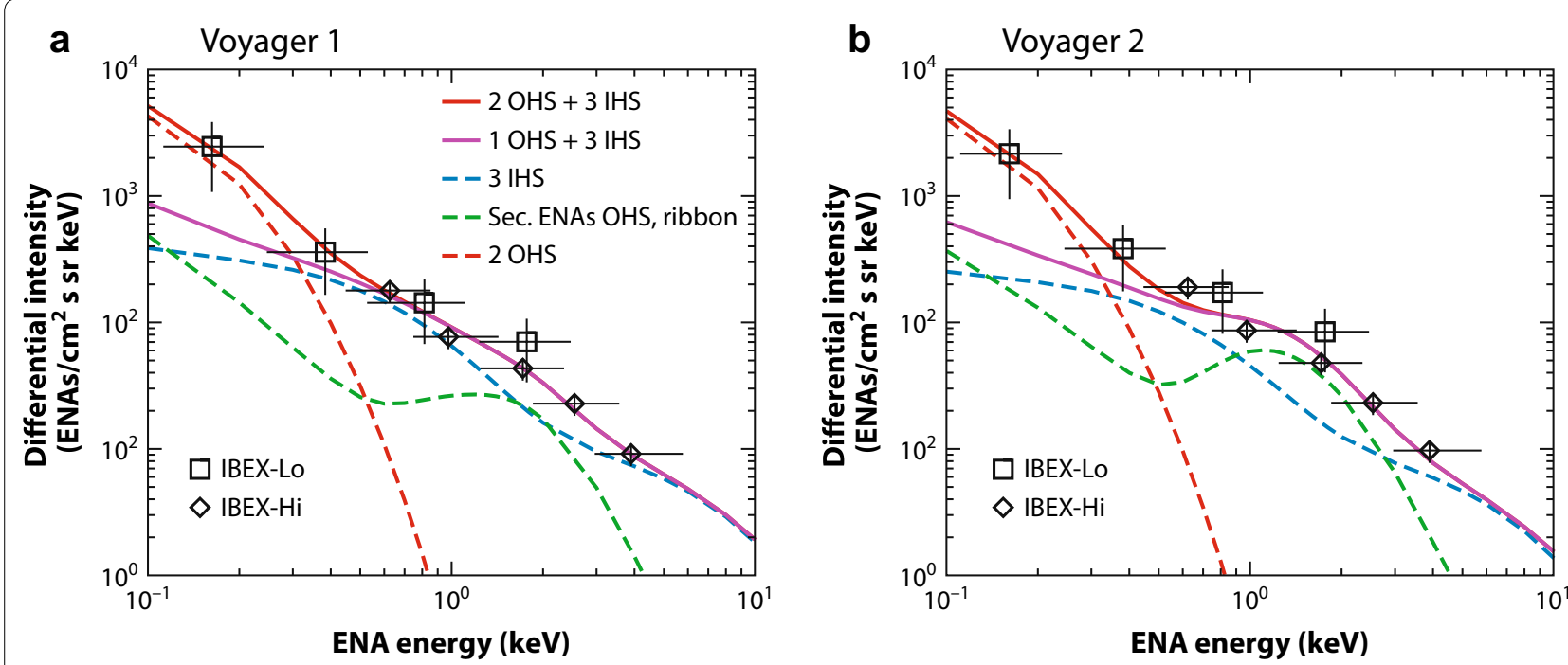

Fig. 3 IBEX-Hi and IBEX-Lo ENA spectra compared with simulations based on one set of parameters considered by Zirnstein et al.- -see text and Zirnstein et al. (2014) for details. Dashed green ENAs from a secondary VLISM population, forming the ribbon; dashed blue three IHS populations with Maxwellian distributions; dashed red ENAs from a hot, PUI VLISM population; solid purple superposition of all three IHS, the secondary ENAs from the ribbon, and a single completely thermalized VLISM population (not visible); solid red superposition of all three IHS, the secondary ENAs from the ribbon, and the two proton VLISM populations (Desai et al. 2014). a The left panel corresponds to observations made by IBEX in the Voyager 1 direction and $\mathbf{b}$ the right panel to observations made in the Voyager 2 direction 
in the model (Zirnstein et al. 2014). Specifically, Zirnstein et al. (1) considered multiple possible sources for OHS PUIs whereas Desai et al. (2014) considered just one case for which the source of OHS PUIs was the IHS, and (2) explored different values for a heating parameter $\alpha$ in their simulations, whereas Desai et al. (2014) assumed a fixed value $\alpha=1 / 4$. The effect of varying these parameters was discussed in detail by Zirnstein et al. (2014), and a similar comparison of the theoretical model and IBEX observations is presented in Fig. 4 of Zirnstein et al. (2014). As illustrated in Fig. 3, below $\sim 0.5 \mathrm{keV}$, the flux is dominated by ENAs from VLISM secondary PUIs, while ENAs from HTS transmitted and reflected PUIs dominate above $0.5 \mathrm{keV}$. Although a small fraction of ENAs from core solar wind protons are visible at $1 \mathrm{AU}$, most exit the HP and become PUIs in the VLISM, producing significant flux near $\sim 0.1 \mathrm{keV}$. Zirnstein et al. (2014) predict that a significant part of the ENA flux seen at $1 \mathrm{AU}$ comes from the VLISM. ENAs created from solar wind PUIs in the VLISM dominate the flux below $\sim 0.2 \mathrm{keV}$, while secondaryinjected, secondary-transmitted, and secondary-reflected PUIs contribute a significant flux up to keV energies, comparable to the flux from the IHS. Our current detailed model (Zirnstein et al. 2014) therefore exploits the properties of PUIs that contribute to heating the VLISM plasma, thereby establishing that not only the low- but also the high-energy flux is a result of the coupling between the IHS and VLISM plasmas through charge exchange. PUIs from the IHS are the source of multiple PUI species in the VLISM. Simulation results (Zirnstein et al. 2014) compare favorably with IBEX data, although perhaps somewhat low at high energies compared to those observed by IBEX since VLISM PUIs created from supersonic solar wind ENAs, or time-dependent solar wind boundary conditions were not included. Nonetheless, these results suggest strong coupling between the IHS and VLISM plasmas through ENA charge exchange, and VLISM PUIs up to $\sim 10 \mathrm{keV}$ may dominate the globally distributed ENA flux visible at 1 AU.

The results from the theoretical models (Zank et al. 2010; Zirnstein et al. 2014) describing the interaction of the solar wind and the partially ionized LISM and the observational results (Desai et al. 2012, 2014) confirm that indeed the IHS and VLISM are multi-component non-equilibrated plasmas. Simplified single-fluid MHD plasma descriptions do not capture the complexity of the plasma. The multicomponent model introduced by Zank et al. (2014) is the first rigorous attempt to extend basic models to incorporate the physics of non-thermal PUI distributions

\section{Modeling a pickup ion-mediated plasma}

The outer heliosphere beyond the ionization cavity (i.e., $\geq \sim 8 \mathrm{AU}$ ) is dominated thermally by PUIs (e.g., Burlaga et al. 1994; Richardson et al. 1995a; Zank 1999; 2015;
Zank et al. 2014). As reported by Decker et al. (2008, 2015), the inner heliosheath pressure contributed by energetic PUIs and anomalous cosmic rays far exceeds that of the thermal background plasma and magnetic field. The VLISM can also be regarded as a multi-component plasma (Desai et al. 2012, 2014; Zirnstein et al. 2014).

Coulomb collisions can equilibrate a background thermal plasma and energetic protons. Assume that the background thermal proton and electron distributions are Maxwellian. If we restrict our attention to PUIs, then they satisfy the ordering $v_{t s} \ll v_{p}<v_{t e}$, where $v_{t s / e}$ denotes the background proton/electron thermal speed respectively and $v_{p}$ the PUI speed. For PUIs experiencing scattering off thermal protons and electrons from a Maxwellian distribution function, the collision frequency between PUIs and protons and PUIs and electrons is given by

$v_{s}^{p s}=\frac{n_{s} e^{4} \ln \Lambda}{2 \pi \varepsilon_{0}^{2} m_{p}^{2} v^{3}} \mathrm{~s}^{-1}, \quad$ and $\quad v_{s}^{p e}=\frac{n_{e} e^{4} \ln \Lambda m_{e}^{1 / 2}}{2(2 \pi)^{3 / 2} \varepsilon_{0}^{2}\left(k T_{e}\right)^{3 / 2} m_{p}} \mathrm{~s}^{-1}$,

respectively. Here $m_{p, e}$ and $n_{p, e}$ denote the proton and electron mass and number density, respectively, $e$ the charge on an electron, $T_{e}$ the electron temperature, $\varepsilon_{0}$ the permittivity of free space, and $\ln \Lambda$ the Coulomb logarithm. If the collisional time scale exceeds the characteristic flow time of the plasma region of interest, $\tau_{f} \simeq L / U$, where $L$ is the size of the region and $U$ the characteristic velocity, then the PUI distribution will not equilibrate with the background thermal plasma. Expressions (1) should be used to determine whether one needs to introduce a plasma model that distinguishes energetic PUIs from background or thermal plasma protons.

Zank et al. (2014) present detailed estimates for the equilibration times for PUIs in the supersonic solar wind of the outer heliosphere, the subsonic solar wind (the inner heliosheath), and the VLSIM using appropriate plasma parameters. In all three regions, the plasma does not equilibrate and cannot therefore be described as a magnetized single-component plasma and at least some elements of a multi-component description are necessary.

PUIs drive streaming instabilities in one form or another, and experience pitch-angle scattering from both self-excited and pre-existing Alfvénic fluctuations. The initial PUI ring-beam distribution is scattered toward isotropy (Lee and Ip 1987; Williams and Zank 1994; Zank 1999; Cannon et al. 2014). Besides pitch-angle scattering by Alfvénic and magnetic field fluctuations, PUIs can experience diffusion in velocity space, both due to counter-propagating Alfvén waves and PUI excited lower hybrid waves, for example. As is typical, we assume that 
pitch-angle scattering is the fastest process associated with wave-particle interactions and neglect velocity diffusion terms. As we show below, pitch-angle scattering serves to introduce both a collisionless heat flux and a non-isotropic pressure tensor into the transport equations describing the PUIs. The pressure tensor modification is expressed as a collisionless viscosity tensor.

To describe a plasma that contains a non-equilibrated PUI population, we construct an appropriate multi-component plasma description for a thermal background plasma comprising electrons and protons and a nonequilibrated PUI component that is subject to pitchangle scattering by turbulence and Alfvénic fluctuations. By making various approximations, we derive successively simpler models. In so doing, we place on a more formal footing the derivation of the well-known two-fluid model of cosmic ray magnetohydrodynamics (Axford et al. 1982; Webb 1983), showing, somewhat unexpectedly and contrary to perceived wisdom, that the cosmic ray number density is in fact included implicitly in the total number density.

\section{The multi-component model}

In deriving a multi-component plasma model that includes PUIs, we shall assume that the distribution functions for the background protons and electrons are each Maxwellian, which ensures the absence of heat flux or stress tensor terms for the background plasma. The exact continuity, momentum, and energy equations governing the thermal electrons $(e)$ and protons $(s)$ are therefore given by

$$
\begin{aligned}
& \frac{\partial n_{e, s}}{\partial t}+\nabla \cdot\left(n_{e, s} \mathbf{u}_{e, s}\right)=0 \\
& m_{e, p} n_{e, p}\left(\frac{\partial \mathbf{u}_{e, s}}{\partial t}+\mathbf{u}_{e, s} \cdot \nabla \mathbf{u}_{e, s}\right) \\
& =-\nabla P_{e, s}+q_{e, s} n_{e, s}\left(\mathbf{E}+\mathbf{u}_{e, s} \times \mathbf{B}\right) \\
& \frac{\partial P_{e, s}}{\partial t}+\mathbf{u}_{e, s} \cdot \nabla P_{e, s}+\gamma_{e, s} P_{e, s} \nabla \cdot \mathbf{u}_{e, s}=0 .
\end{aligned}
$$

Here $n_{e, s}, \mathbf{u}_{e, s}$, and $P_{e, s}$ are the macroscopic fluid variables for the electron/proton number density, velocity, and pressure, respectively, $\gamma_{e, s}$ the electron/proton adiabatic index, $\mathbf{E}$ the electric field, $\mathbf{B}$ the magnetic field, and $q_{e, s}$ the charge of particle.

The streaming instability for the unstable PUI ringbeam distribution excites Alfvénic fluctuations. The self-generated fluctuations and in situ turbulence serve to scatter PUIs in pitch-angle. The Alfvén waves and magnetic field fluctuations both propagate and convect with the bulk velocity of the system
$\mathbf{U}=\mathbf{U}\left(\mathbf{u}_{e}, \mathbf{u}_{s}, \mathbf{u}_{p}, n_{e}, n_{s}, n_{p}, m_{e}, m_{p}\right)$, where $n_{p}$ and $\mathbf{u}_{p}$ refer to PUI variables. The PUIs are governed by the Boltzmann transport equation with a collisional term $\delta f /\left.\delta t\right|_{\mathcal{c}}$,

$$
\frac{\partial f}{\partial t}+\mathbf{v} \cdot \nabla f+\frac{e}{m_{p}}(\mathbf{E}+\mathbf{v} \times \mathbf{B}) \cdot \nabla_{v} f=\left.\frac{\delta f}{\delta t}\right|_{c},
$$

for average electric and magnetic fields $\mathbf{E}$ and $\mathbf{B}$. On transforming the transport equation (5) into a frame that ensures there is no change in PUI momentum and energy due to scattering, assuming that the cross-helicity is zero, and introducing the random velocity $\mathbf{c}=\mathbf{v}-\mathbf{U}$, we obtain

$$
\begin{gathered}
\frac{\partial f}{\partial t}+\left(U_{i}+c_{i}\right) \frac{\partial f}{\partial x_{i}}+\left[\frac{e}{m_{p}}(\mathbf{E}+\mathbf{U} \times \mathbf{B})_{i}+\frac{e}{m_{p}}(\mathbf{c} \times \mathbf{B})_{i}\right. \\
\left.-\frac{\partial U_{i}}{\partial t}-\left(U_{j}+c_{j}\right) \frac{\partial U_{i}}{\partial x_{j}}\right] \frac{\partial f}{\partial c_{i}}=\left.\frac{\delta f}{\delta t}\right|_{c}
\end{gathered}
$$

The velocity $\mathbf{U}$ is still unspecified so we choose $\mathbf{U}$ such that $\mathbf{E}^{\prime} \equiv \mathbf{E}+\mathbf{U} \times \mathbf{B}=0$. This assumption corresponds to choosing

$$
\mathbf{U}_{\perp}=\mathbf{U}-\mathbf{U}_{\|}=\frac{\mathbf{E} \times \mathbf{B}}{B^{2}} \equiv \mathbf{U},
$$

since we choose $\mathbf{U}_{\|}=0\left(\mathbf{U}_{\|}\right.$is parallel to $\mathbf{B}$ and therefore arbitrary). The use of the velocity $\mathbf{U}$ then yields

$$
\begin{aligned}
& \frac{\partial f}{\partial t}+\left(U_{i}+c_{i}\right) \frac{\partial f}{\partial x_{i}}+\left[\frac{e}{m_{p}}(\mathbf{c} \times \mathbf{B})_{i}\right. \\
& \left.-\frac{\partial U_{i}}{\partial t}-\left(U_{j}+c_{j}\right) \frac{\partial U_{i}}{\partial x_{j}}\right] \frac{\partial f}{\partial c_{i}}=\left.\frac{\delta f}{\delta t}\right|_{c} .
\end{aligned}
$$

By taking moments of (8), we can derive the evolution equations for the macroscopic PUI variables, such as the number density $n_{p}=\int f d^{3} c$, momentum density $n_{p} u_{p_{i}}=\int c_{i} f d^{3} c$, and energy density. Moments of the collisional term $\delta f /\left.\delta t\right|_{c}$ are zero. The zeroth moment of (8) yields the continuity equation for PUIs,

$$
\frac{\partial n_{p}}{\partial t}+\frac{\partial}{\partial x_{i}}\left(n_{p}\left(U_{i}+u_{p_{i}}\right)\right)=0,
$$

where $\mathbf{u}_{p}$ is the PUI bulk velocity in the guiding center frame. For the first moment, we multiply (8) by $c_{j}$ and integrate over velocity space. This yields, after a little algebra, the momentum equation for PUIs,

$$
\begin{aligned}
& \frac{\partial}{\partial t}\left(n_{p}\left(U_{j}+u_{p_{j}}\right)\right)+\nabla \cdot\left[n_{p} \mathbf{U}\left(U_{j}+u_{p_{j}}\right)+n_{p} \mathbf{u}_{p} U_{j}\right] \\
& \quad+\frac{\partial}{\partial x_{i}} \int c_{i} c_{j} f d^{3} c=\frac{e}{m_{p}} n_{p} \varepsilon_{j k l} u_{p_{k}} B_{l}
\end{aligned}
$$

where $\varepsilon_{i j k}$ is the Levi-Civeta tensor. Note the presence of the term $\int c_{i} c_{j} f d^{3} c$, which is the momentum flux or pressure tensor. 
To close Eq. (10), we need to evaluate the momentum flux, which requires that we solve (8) for the PUI distribution function $f$. In solving (8), we assume (1) that the PUI distribution is gyrotropic, and (2) that scattering of PUIs is sufficiently rapid to ensure that the PUI distribution is nearly isotropic. We can therefore average (8) over gyrophase, obtaining the "focused transport equation" for non-relativistic particles (Isenberg 1997). Details of the derivation can be found in Ch. 5 of Zank (2014). To solve the gyrophase-averaged transport equation requires that we specify the scattering or collisional operator. We make the simplest possible choice, which is the isotropic pitchangle diffusion operator,

$$
\frac{\partial}{\partial \mu}\left(v_{s}\left(1-\mu^{2}\right) \frac{\partial f}{\partial \mu}\right),
$$

where $\mu=\cos \theta$ is the cosine of the particle pitch-angle $\theta$, and $v_{s}=\tau_{s}^{-1}$ is the scattering frequency. The form of the scattering operator (11) allows us to solve the focused transport equation using a Legendre polynomial expansion of the distribution function $f$. The second-order correct solution to the gyrophase-averaged form of Eq. (8) is

$$
\begin{aligned}
& f \simeq f_{0}+\mu f_{1}+\frac{1}{2}\left(3 \mu^{2}-1\right) f_{2} ; \\
& f_{0}=f_{0}(\mathbf{x}, c, t) ; \\
& f_{1}=-\frac{c \tau_{s}}{3} b_{i} \frac{\partial f_{0}}{\partial x_{i}}+\frac{D U_{i}}{D t} \frac{\tau_{s}}{3} b_{i} \frac{\partial f_{0}}{\partial c} ; \\
& f_{2} \simeq \frac{c \tau_{s}}{15}\left(b_{i} b_{j} \frac{\partial U_{j}}{\partial x_{i}}-\frac{1}{3} \frac{\partial U_{i}}{\partial x_{i}}\right) \frac{\partial f_{0}}{\partial c},
\end{aligned}
$$

where $c=|\mathbf{c}|$ is the particle random speed, $\mathbf{b} \equiv \mathbf{B} / B$ is a directional unit vector defined by the magnetic field, and $D / D t \equiv \partial / \partial t+U_{i} \partial / \partial x_{i}$ is the convective derivative. The expansion terms $f_{0}, f_{1}$ and $f_{2}$ are functions of position, time, and particle random speed $c$, i.e., independent of $\mu$ (and of course gyrophase $\phi$ ). Of particular importance is the retention of the large-scale acceleration, and shear terms. These terms are often neglected in the derivation of the transport equation describing $f_{0}$ (for relativistic particles, the transport equation is the familiar cosmic ray transport equation). In deriving a multi-fluid model, retaining the various flow velocity terms is essential to derive the correct multi-fluid formulation for PUIs. We need to evaluate

$$
\begin{aligned}
\int c_{i} c_{j} f d^{3} c & =\int\left(c_{i}-u_{p_{i}}\right)\left(c_{j}-u_{p_{j}}\right) f d^{3} c+n_{p} u_{p_{i}} u_{p_{j}} \\
& \equiv \int c_{i}^{\prime} c_{j}^{\prime} f d^{3} c+n_{p} u_{p_{i}} u_{p_{j}} \\
& \simeq \int c_{i}^{\prime} c_{j}^{\prime}\left(f_{0}+\mu f_{1}+\frac{1}{2}\left(3 \mu^{2}-1\right) f_{2}\right) d^{3} c^{\prime}+n_{p} u_{p_{i}} u_{p_{j}},
\end{aligned}
$$

from which we find the zeroth- and first-order expressions,

$$
\begin{aligned}
\int c_{i}^{\prime} c_{j}^{\prime} f_{0} d^{3} c & =\frac{1}{m_{p}}\left(\delta_{i j} P_{p}\right), \quad \int c_{i}^{\prime} c_{j}^{\prime} \mu f_{1} d^{3} c=0, \\
P_{p} & \equiv m_{p} \frac{4 \pi}{3} \int c^{\prime 2} f_{0} c^{\prime 2} d c .
\end{aligned}
$$

Consequently, the first-order PUI stress tensor is identically zero and the pressure is isotropic, $\delta_{i j} P_{p}$.

The inclusion of the second-order terms yields a nonzero collisionless stress tensor. Since the PUI pressure is defined in the frame of the bulk PUI velocity $\mathbf{u}_{p}$, the distribution function over which the integral is taken needs to be evaluated in this frame. Since the expression (15) for $f_{2}$ is a function of the guiding center velocity $\mathbf{U}$, we need to transform to the frame $\mathbf{U}_{p}=\mathbf{U}+\mathbf{u}_{p}$. On using the solution (15) for $f_{2}$, we obtain

$$
\begin{aligned}
& \int c_{x}^{\prime 2} \frac{1}{2}\left(3 \mu^{2}-1\right) f_{2} d^{3} c^{\prime}=\int c_{y}^{\prime 2} \frac{1}{2}\left(3 \mu^{2}-1\right) f_{2} d^{3} c^{\prime} \\
&=\frac{\eta}{15}\left(b_{i} b_{j} \frac{\partial U_{p j}}{\partial x_{i}}-\frac{1}{3} \frac{\partial U_{p i}}{\partial x_{i}}\right) \\
& \int c_{z}^{\prime 2} \frac{1}{2}\left(3 \mu^{2}-1\right) f_{2} d^{3} c^{\prime}=-\frac{2 \eta}{15}\left(b_{i} b_{j} \frac{\partial U_{p j}}{\partial x_{i}}-\frac{1}{3} \frac{\partial U_{p i}}{\partial x_{i}}\right) \\
& \int c_{i}^{\prime} c_{j}^{\prime} \frac{1}{2}\left(3 \mu^{2}-1\right) f_{2} d^{3} c^{\prime}=0, \quad(i \neq j),
\end{aligned}
$$

where the coefficient of viscosity $\eta$ is defined as

$\eta \equiv \frac{4 \pi}{15} \int \frac{\partial}{\partial c^{\prime}}\left(c^{\prime 4} c \tau_{s}\right) f_{0} d c^{\prime} \simeq \frac{4 \pi}{3} \int c^{\prime 2} \tau_{s} f_{0} c^{\prime 2} d c^{\prime} \simeq \frac{P_{p} \tau_{s}}{m_{p}}$.

The first equality in (20) is the formal definition of the coefficient of viscosity for the PUI gas. If we assume (probably reasonably) that $|\mathbf{c}| \gg\left|\mathbf{u}_{p}\right|$, then we obtain the second equality, which may be regarded as a PUI pressure moment weighted by the PUI scattering time. Finally, if we assume that $\tau_{s}$ is independent of $c$, we then obtain the "classical" form (20) of the viscosity coefficient. The pressure tensor may therefore be expressed as

$$
\left(P_{i j}\right)=P_{p}\left(\delta_{i j}\right)+\left(\begin{array}{lll}
1 & 0 & 0 \\
0 & 1 & 0 \\
0 & 0 & -2
\end{array}\right) \frac{\eta}{15}\left(b_{k} b_{\ell} \frac{\partial U_{p k}}{\partial x_{\ell}}-\frac{1}{3} \frac{\partial U_{p m}}{\partial x_{m}}\right) .
$$

If we introduce a "viscosity matrix,"

$$
\left(M_{k \ell}\right) \equiv\left(\eta_{k \ell}\right)=\left(\frac{\eta}{15} b_{k} b_{\ell}\right) \simeq\left(\frac{1}{15} \frac{P_{p} \tau_{s} b_{k} b_{\ell}}{m_{p}}\right),
$$


and note that $\eta_{i j}=\eta_{j i}$ and $\eta / 15=\eta_{11}+\eta_{22}+\eta_{33}=\eta_{i j} \delta_{i j}$ (since $b^{2}=1$ ), we can rewrite (21) in the more revealing "classical" stress tensor form,

$$
\begin{aligned}
& \frac{\eta}{15}\left(b_{k} b_{\ell} \frac{\partial U_{p k}}{\partial x_{\ell}}-\frac{1}{3} \frac{\partial U_{p m}}{\partial x_{m}}\right) \\
& \quad=\frac{\eta_{k \ell}}{2}\left(\frac{\partial U_{p k}}{\partial x_{\ell}}+\frac{\partial U_{p \ell}}{\partial x_{k}}\right)-\frac{1}{3} \eta_{k \ell} \delta_{k \ell} \frac{\partial U_{p m}}{\partial x_{m}} \\
& \quad=\frac{\eta_{k \ell}}{2}\left(\frac{\partial U_{p k}}{\partial x_{\ell}}+\frac{\partial U_{p \ell}}{\partial x_{k}}-\frac{2}{3} \delta_{k \ell} \frac{\partial U_{p m}}{\partial x_{m}}\right) .
\end{aligned}
$$

The pressure tensor is therefore the sum of an isotropic scalar pressure $P_{p}$ associated with drift and curvatur and the stress tensor, i.e.,

$$
\begin{aligned}
\left(P_{i j}\right) & =P_{p}\left(\delta_{i j}\right)+\left(\begin{array}{ccc}
1 & 0 & 0 \\
0 & 1 & 0 \\
0 & 0 & -2
\end{array}\right) \frac{\eta_{k \ell}}{2}\left(\frac{\partial U_{p k}}{\partial x_{\ell}}+\frac{\partial U_{p \ell}}{\partial x_{k}}-\frac{2}{3} \delta_{k \ell} \frac{\partial U_{p m}}{\partial x_{m}}\right) \\
& \equiv P_{p} \mathbf{I}+\Pi_{p} .
\end{aligned}
$$

The stress tensor is a generalization of the "classical" form in that several coefficients of viscosity are present, and of course the derivation here is for a collisionless charged gas of PUIs experiencing only pitch-angle scattering by turbulent magnetic fluctuations. Use of the pressure tensor (24) yields a "Navier-Stokes"-like modification of the PUI momentum equation,

$$
\begin{aligned}
& \frac{\partial}{\partial t}\left(\rho_{p} \mathbf{U}_{p}\right)+\nabla \cdot\left[\rho_{p} \mathbf{U}_{p} \mathbf{U}_{p}+\mathbf{I} P_{p}\right]=e n_{p}\left(\mathbf{E}+\mathbf{U}_{p} \times \mathbf{B}\right) \\
& -\nabla \cdot\left(\begin{array}{ccc}
1 & 0 & 0 \\
0 & 1 & 0 \\
0 & 0 & -2
\end{array}\right) \frac{\eta_{k \ell}}{2}\left(\frac{\partial U_{p_{k}}}{\partial x_{\ell}}+\frac{\partial U_{p_{\ell}}}{\partial x_{k}}-\frac{2}{3} \delta_{k \ell} \frac{\partial U_{p_{m}}}{\partial x_{m}}\right), \\
& \quad=e n_{p}\left(\mathbf{E}+\mathbf{U}_{p} \times \mathbf{B}\right)-\nabla \cdot \Pi_{p}
\end{aligned}
$$

where we used the transformation $\mathbf{U}_{p}=\mathbf{u}_{p}+\mathbf{U}$ for the remaining velocity terms in (10) and $\rho_{p}=m_{p} n_{p}$.

If we introduce $\mathbf{c}^{\prime} \equiv \mathbf{c}-\mathbf{u}_{p}$ as before, we can express the heat flux $\mathbf{q}(\mathbf{x}, t)$ through the definition

$$
\begin{aligned}
q_{i}(\mathbf{x}, t) \equiv & m_{p} \int \frac{1}{2} c^{\prime 2} c_{j}^{\prime} f d^{3} c^{\prime}=\frac{m_{p}}{2} \int c^{2} c_{i} f d^{3} c \\
& -\frac{5}{2} u_{p_{i}} P_{p}-\frac{1}{2} \rho_{p} u_{p}^{2} u_{p_{i}} .
\end{aligned}
$$

The equation for the total energy of the PUIs can then be derived from (8), yielding

$$
\begin{aligned}
& \frac{\partial}{\partial t}\left(\frac{1}{2} \rho_{p} U_{p}^{2}+\frac{3}{2} P_{p}\right)+\frac{\partial}{\partial x_{i}}\left[\frac{1}{2} \rho_{p} U_{p}^{2} U_{p_{i}}+\frac{5}{2} P_{p} U_{p_{i}}+\Pi_{i j} U_{p_{j}}+q_{i}\right] \\
& \quad=e n_{p} U_{p_{i}}\left(E_{i}+\left(\mathbf{U}_{p} \times \mathbf{B}\right)_{i}\right),
\end{aligned}
$$

after transforming to $\mathbf{U}_{p}$. To evaluate the heat flux, we have

$\frac{1}{2} \int c^{\prime 2} c_{i}^{\prime} f_{0} d^{3} c=\pi \int c^{\prime 3} \mu b_{i} f_{0} c^{\prime 2} d c^{\prime}=0$,

and

$$
\begin{aligned}
\frac{m_{p}}{2} \int c^{\prime 2} c_{i}^{\prime} \mu f_{1} d^{3} c^{\prime} & =-\frac{2 \pi}{3} m_{p} \int c^{\prime 2} \kappa_{i j} \frac{\partial f_{0}}{\partial x_{j}} c^{\prime 2} d c^{\prime} \\
& =-\frac{1}{2} \bar{\kappa}_{i j} \frac{\partial P_{p}}{\partial x_{j}}=q_{i}(\mathbf{x}, t) .
\end{aligned}
$$

In (28), we introduced the spatial diffusion coefficient

$$
\kappa_{i j} \equiv b_{i} \frac{c^{2} \tau_{s}}{3} b_{j},
$$

together with PUI speed-averaged form $\bar{\kappa}_{i j} \equiv K_{i j}$. The collisionless heat flux for PUIs is therefore described in terms of the PUi pressure gradient and consequently the averaged spatial diffusion introduces a PUI diffusion time and length scale into the multi-fluid system.

For continuous flows, the transport equation for the PUI pressure $P_{p}$ can be derived from (27), yielding

$\frac{\partial P_{p}}{\partial t}+U_{p_{i}} \frac{\partial P_{p}}{\partial x_{i}}+\frac{5}{3} P_{p} \frac{\partial U_{p_{i}}}{\partial x_{i}}=\frac{1}{3} \frac{\partial}{\partial x_{i}}\left(K_{i j} \frac{\partial P_{p}}{\partial x_{j}}\right)-\frac{2}{3} \Pi_{i j} \frac{\partial U_{p_{j}}}{\partial x_{i}}$,

illustrating that the PUI heat flux yields a spatial diffusion term in the PUI equation of state together with a viscous dissipation term. The PUI system of equations is properly closed and correct to the second-order. Note the typo in Zank et al. (2014) since we mistakenly omitted the viscous term of Eq. (30) in the corresponding pressure equation.

The full system of PUI equations can be written in the form

$$
\begin{gathered}
\frac{\partial \rho_{p}}{\partial t}+\nabla \cdot\left(\rho_{p} \mathbf{U}_{p}\right)=0 \\
\frac{\partial}{\partial t}\left(\rho_{p} \mathbf{U}_{p}\right)+\nabla \cdot\left[\rho_{p} \mathbf{U}_{p} \mathbf{U}_{p}+\mathbf{I} P_{p}+\Pi\right]=e n_{p}\left(\mathbf{E}+\mathbf{U}_{p} \times \mathbf{B}\right)
\end{gathered}
$$

$$
\begin{aligned}
\frac{\partial}{\partial t}( & \left.\frac{1}{2} \rho_{p} U_{p}^{2}+\frac{3}{2} P_{p}\right)+\nabla \cdot\left[\frac{1}{2} \rho_{p} U_{p}^{2} \mathbf{U}_{p}\right. \\
& \left.+\frac{5}{2} P_{p} \mathbf{U}_{p}+\Pi \cdot \mathbf{U}_{p}-\frac{1}{2} \mathbf{K} \cdot \nabla P_{p}\right]=e n_{p} \mathbf{U}_{p} \cdot \mathbf{E},
\end{aligned}
$$

which is the form we use below. 
The full thermal electron-thermal proton-PUI multifluid system is therefore given by Eqs. (2)-(4) and (31)(33) or (30), together with Maxwell's equations,

$$
\begin{aligned}
& \frac{\partial \mathbf{B}}{\partial t}=-\nabla \times \mathbf{E} ; \\
& \nabla \times \mathbf{B}=\mu_{0} \mathbf{J} ; \\
& \nabla \cdot \mathbf{B}=0 ; \\
& \mathbf{J}=e\left(n_{s} \mathbf{u}_{s}+n_{p} \mathbf{U}_{p}-n_{e} \mathbf{u}_{e}\right),
\end{aligned}
$$

where $\mathbf{J}$ is the current and $\mu_{0}$ the permeability of free space. The diffusion tensor is assumed to be of a simple diagonal form (i.e., we do not include the off-diagonal terms associated with drift and curvature-see the discussion in Zank (2014) and we specify

$\mathbf{K}=\left(\begin{array}{ccc}\kappa_{\perp} & 0 & 0 \\ 0 & \kappa_{\perp} & 0 \\ 0 & 0 & \kappa_{\|}\end{array}\right) ; \quad \kappa_{\perp}=\eta \frac{1}{3 \Omega_{p}} C_{0}^{2}, \quad \kappa_{\|}=\frac{1}{3 \Omega_{p}} C_{0}^{2}$.

We parametrize the perpendicular component of the heat conduction tensor by a term $\eta<1$. In estimating the diffusion coefficients (38) from (29), we choose a characteristic PUI speed for the region of interest and assume that the scattering time can be approximated by a time scale greater than the corresponding gyroperiod.

\section{Single-fluid-like model}

For many problems, the complete multi-component model derived above is far too complicated to solve. The multi-fluid system (2)-(4) and (31)-(33) or (30), together with Maxwell's equations can be considerably reduced in complexity by making the key assumption that $\mathbf{U}_{p} \simeq \mathbf{u}_{s}$. The assumption that $\mathbf{U}_{p} \simeq \mathbf{u}_{s}$ is quite reasonable since (i) the bulk flow velocity of the plasma is dominated by the background protons since the PUI component scatters off fluctuations moving with the background plasma speed and (ii) the large-scale motional electric field forces newly created PUIs to essentially co-move with the background plasma flow perpendicular to the mean magnetic field. Accordingly, we let $\mathbf{U}_{p} \simeq \mathbf{u}_{s}=\mathbf{U}_{i}$ be the bulk proton (i.e., thermal background protons and PUIs) velocity. The thermal proton and PUI continuity and momentum equations are therefore trivially combined as

$$
\begin{aligned}
& \frac{\partial n_{i}}{\partial t}+\nabla \cdot\left(n_{i} \mathbf{U}_{i}\right)=0 ; \\
& m_{p} n_{i}\left(\frac{\partial \mathbf{U}_{i}}{\partial t}+\mathbf{U}_{i} \cdot \nabla \mathbf{U}_{i}\right)=-\nabla\left(P_{s}+P_{p}\right) \\
& \quad+e n_{i}\left(\mathbf{E}+\mathbf{U}_{i} \times \mathbf{B}\right)-\nabla \cdot \Pi_{p},
\end{aligned}
$$

where $n_{i}=n_{s}+n_{p}$. Since the PUIs are not thermally equilibrated with the background plasma $\left(T_{s} \neq T_{p}\right)$, we need to deal separately with the $P_{s}$ and $P_{p}$ equations. These become

$$
\begin{gathered}
\frac{\partial P_{s}}{\partial t}+\mathbf{U}_{i} \cdot \nabla P_{s}+\gamma_{s} P_{s} \nabla \cdot \mathbf{U}_{i}=0 \\
\frac{\partial P_{p}}{\partial t}+U_{i} \frac{\partial P_{p}}{\partial x_{i}}+\frac{5}{3} P_{p} \frac{\partial U_{i}}{\partial x_{i}}=\frac{1}{3} \frac{\partial}{\partial x_{i}}\left(K_{i j} \frac{\partial P_{p}}{\partial x_{j}}\right)-\frac{2}{3} \Pi_{i j} \frac{\partial U_{j}}{\partial x_{i}} .
\end{gathered}
$$

We can combine the proton Eqs. (39)-(42) with the electron Eqs. (2)-(4) to obtain an MHD-like system of equations. On defining the macroscopic variables,

$$
\begin{aligned}
& \rho \equiv m_{e} n_{e}+m_{p} n_{i} ; \quad q \equiv-e\left(n_{e}-n_{i}\right) ; \\
& \quad \rho \mathbf{U} \equiv m_{e} n_{e} \mathbf{u}_{e}+m_{p} n_{i} \mathbf{U}_{i} ; \mathbf{J} \equiv-e\left(n_{e} \mathbf{u}_{e}-n_{i} \mathbf{U}_{i}\right),
\end{aligned}
$$

we can express

$$
\begin{aligned}
& n_{e}=\frac{\rho-\left(m_{p} / e\right) q}{m_{p}(1-\xi)} \simeq \rho / m_{p} ; \quad n_{i}=\frac{\rho+\xi\left(m_{p} / e\right) q}{m_{p}(1+\xi)} \simeq \rho / m_{p} ; \\
& \mathbf{u}_{e}=\frac{\rho \mathbf{U}-\left(m_{p} / e\right) \mathbf{J}}{\rho-\left(m_{p} / e\right) q} \simeq \mathbf{U}-\frac{m_{p}}{e} \frac{\mathbf{J}}{\rho} ; \quad \mathbf{u}_{i}=\frac{\rho \mathbf{U}+\xi\left(m_{p} / e\right) \mathbf{J}}{\rho+\xi\left(m_{p} / e\right) q} \simeq \mathbf{U}
\end{aligned}
$$

where the smallness of the mass ratio $\xi \equiv m_{e} / m_{p} \ll 1$ has been exploited. Use of the approximations (44) allows us to combine the continuity and momentum equations in the usual way and to rewrite the thermal electron and proton pressure in terms of the single-fluid macroscopic variables. Thus,

$$
\frac{\partial \rho}{\partial t}+\nabla \cdot(\rho \mathbf{U})=0
$$

$\rho\left(\frac{\partial \mathbf{U}}{\partial t}+\mathbf{U} \cdot \nabla \mathbf{U}\right)=-\nabla\left(P_{e}+P_{s}+P_{p}\right)+\mathbf{J} \times \mathbf{B}-\nabla \cdot \Pi ;$

$$
\begin{aligned}
& \frac{\partial P_{s}}{\partial t}+\mathbf{U} \cdot \nabla P_{s}+\gamma_{s} P_{s} \nabla \cdot \mathbf{U}=0 \\
& \frac{\partial P_{e}}{\partial t}+\mathbf{U} \cdot \nabla P_{e}+\gamma_{e} P_{e} \nabla \cdot \mathbf{U}=\frac{m_{p}}{e \rho} \mathbf{J} \cdot \nabla P_{e}+\frac{\gamma_{e} m_{p}}{e} P_{e} \nabla \cdot\left(\frac{\mathbf{J}}{\rho}\right),
\end{aligned}
$$

where

$\Pi_{k \ell}=\left(\begin{array}{ccc}1 & 0 & 0 \\ 0 & 1 & 0 \\ 0 & 0 & -2\end{array}\right) \frac{\eta_{k \ell}}{2}\left(\frac{\partial U_{k}}{\partial x_{\ell}}+\frac{\partial U_{\ell}}{\partial x_{k}}-\frac{2}{3} \delta_{k \ell} \frac{\partial U_{m}}{\partial x_{m}}\right)$.

Since we may assume that the current density is much less than the momentum flux, i.e., $|\mathbf{J}| \ll|\rho \mathbf{U}|$, we can simplify (48) further by neglecting the RHS. By assuming 
that $\gamma_{e}=\gamma_{s}=\gamma$, we can combine the thermal proton and electron equations in a single thermal plasma pressure equation with $P \equiv P_{e}+P_{s}$,

$$
\frac{\partial P}{\partial t}+\mathbf{U} \cdot \nabla P+\gamma P \nabla \cdot \mathbf{U}=0 .
$$

Note that at this point, no assumptions about either the thermal electron or proton pressures (or temperatures) have been made.

Finally, we need an equation for the electric field $\mathbf{E}$. To do so, we multiply the respective momentum equations by the electron or proton charge, sum, and use the approximations (44) to obtain

$$
\begin{gathered}
\xi\left(\frac{m_{p}}{e}\right)^{2} \frac{1}{\rho}\left[\frac{\partial \mathbf{J}}{\partial t}+\nabla \cdot(\mathbf{J U}+\mathbf{U J})\right] \\
=\frac{m_{p}}{e \rho}\left(\nabla P_{e}-\mathbf{J} \times \mathbf{B}-\xi \nabla\left(P_{s}+P_{p}\right)\right. \\
-\xi \nabla \cdot \Pi)+\mathbf{E}+\mathbf{U} \times \mathbf{B} .
\end{gathered}
$$

The generalized Ohm's law is therefore

$$
\mathbf{E}=-\mathbf{U} \times \mathbf{B}-\frac{m_{p}}{e \rho}\left(\nabla P_{e}-\mathbf{J} \times \mathbf{B}-\xi \nabla P_{p}\right),
$$

where we have retained the PUI pressure since in principle it can be a high-temperature component of the plasma system and $\xi P_{p}$ may be comparable to the $P_{e}$ term. For typical cases of interest, however, the $P_{p}$ term can be neglected in Ohm's law (50). Neglect of the electron pressure and Hall current term then yields the usual form of Ohm's law.

The reduced single-fluid model equations may therefore be summarized as

$$
\begin{gathered}
\frac{\partial \rho}{\partial t}+\nabla \cdot(\rho \mathbf{U})=0 ; \\
\rho\left(\frac{\partial \mathbf{U}}{\partial t}+\mathbf{U} \cdot \nabla \mathbf{U}\right)=-\nabla\left(P+P_{p}\right)+\mathbf{J} \times \mathbf{B}-\nabla \cdot \Pi ;(52) \\
\frac{\partial}{\partial t}\left(\frac{1}{2} \rho U^{2}+\frac{3}{2}\left(P+P_{p}\right)+\frac{1}{2 \mu_{0}} B^{2}\right)+\nabla \cdot\left[\frac{1}{2} \rho U^{2} \mathbf{U}+\frac{5}{2}\left(P+P_{p}\right) \mathbf{U}\right. \\
\left.+\frac{1}{\mu_{0}} B^{2} \mathbf{U}-\frac{1}{\mu_{0}} \mathbf{U} \cdot \mathbf{B B}+\Pi \cdot \mathbf{U}_{p}-\frac{1}{2} \mathbf{K} \cdot \nabla P_{p}\right]=0 ; \\
\quad \frac{\partial P}{\partial t}+\mathbf{U} \cdot \nabla P+\gamma P \nabla \cdot \mathbf{U}=0 ; \\
\mathbf{E}=-\mathbf{U} \times \mathbf{B} ; \quad \frac{\partial \mathbf{B}}{\partial t}=-\nabla \times \mathbf{E} ; \quad \mu_{0} \mathbf{J}=\nabla \times \mathbf{B} ; \quad \nabla \cdot \mathbf{B}=0 .
\end{gathered}
$$

The single-fluid description (51)-(55) differs from the standard MHD model in that a separate description for the PUI pressure is required. Instead of the conservation of energy Eq. (53), one could use the PUI pressure Eq. (42) for continuous flows. PUIs introduce both a collisionless heat conduction and viscosity into the system.

The model Eqs. (51)-(55), despite being appropriate to non-relativistic PUIs, are identical to the so-called twofluid MHD system of equations used to describe cosmic ray-mediated plasmas (Webb 1983). However, the derivation of the two models is substantially different in that the cosmic ray number density is explicitly neglected in the two-fluid cosmic ray model and a Chapman-Enskog derivation is not used in deriving the cosmic ray hydrodynamic equations. Nonetheless, the sets of equations that emerge are the same indicating that the cosmic ray two-fluid equations do in fact include the cosmic ray number density explicitly.

The single-fluid-like model may be extended to include, e.g., anomalous cosmic rays (ACRs) as well as PUIs. In this case, the ACRs are relativistic particles. The same analysis carries over, and one has an obvious extension of the model Eqs. (51)-(55) with the inclusion of the ACR pressure. Thus, the extension of (51)-(55) is

$$
\begin{aligned}
\frac{\partial \rho}{\partial t}+\nabla \cdot(\rho \mathbf{U})=0 & \\
\rho\left(\frac{\partial \mathbf{U}}{\partial t}+\mathbf{U} \cdot \nabla \mathbf{U}\right)= & -\nabla\left(P+P_{p}+P_{A}\right)+\mathbf{J} \times \mathbf{B} \\
& -\nabla \cdot \Pi_{p}-\nabla \cdot \Pi_{A}
\end{aligned}
$$

$$
\frac{\partial P}{\partial t}+\mathbf{U} \cdot \nabla P+\gamma P \nabla \cdot \mathbf{U}=0
$$

$$
\begin{aligned}
& \frac{\partial P_{p}}{\partial t}+\mathbf{U} \cdot \nabla P_{p}+\gamma_{p} P_{p} \nabla \cdot \mathbf{U} \\
& \quad=\frac{1}{3} \nabla \cdot\left(\mathbf{K}_{\mathbf{p}} \cdot \nabla P_{p}\right)-\left(\gamma_{p}-1\right) \Pi_{p}:(\nabla \mathbf{U}) ;
\end{aligned}
$$

$$
\begin{aligned}
& \frac{\partial P_{A}}{\partial t}+\mathbf{U} \cdot \nabla P_{A}+\gamma_{A} P_{A} \nabla \cdot \mathbf{U} \\
& \quad=\frac{1}{3} \nabla \cdot\left(\mathbf{K}_{\mathbf{A}} \cdot \nabla P_{A}\right)-\left(\gamma_{A}-1\right) \Pi_{A}:(\nabla \mathbf{U}) ; \\
& \mathbf{E}=-\mathbf{U} \times \mathbf{B} ; \quad \frac{\partial \mathbf{B}}{\partial t}=-\nabla \times \mathbf{E} ; \quad \mu_{0} \mathbf{J}=\nabla \times \mathbf{B} ; \quad \nabla \cdot \mathbf{B}=0,
\end{aligned}
$$

where we have introduced the ACR pressure $P_{A}$, the corresponding stress tensor $\Pi_{A}$, the ACR diffusion tensor $\mathbf{K}_{A}$ and adiabatic index $\gamma_{A}\left(4 / 3 \leq \gamma_{A} \leq 5 / 3\right)$. The coupled system (56)-(61) is the simplest continuum model to describe a non-equilibrated plasma comprising a thermal proton-electron plasma with suprathermal 
particles (e.g., PUIs or even solar energetic particles) and relativistic energy (anomalous) cosmic rays. The system includes both the collisionless heat flux and viscosity associated with the suprathermal and relativistic particle distributions.

On reverting to Eqs. (51)-(55), we can recover the standard form of the MHD equations if we set the heat conduction spatial diffusion tensor $\mathbf{K}=0$ and the coefficient of viscosity $\left(\eta_{k l}\right)=0$, which corresponds to assuming $\tau_{s} \rightarrow 0$. If the total thermodynamic pressure $P_{\text {total }}=P+P_{p}$ is introduced, then we recover the standard MHD equations (dropping the subscript "total"), i.e.,

$$
\begin{gathered}
\frac{\partial \rho}{\partial t}+\nabla \cdot(\rho \mathbf{U})=0 ; \\
\rho \frac{\partial \mathbf{U}}{\partial t}+\rho \mathbf{U} \cdot \nabla \mathbf{U}+(\gamma-1) \nabla e+(\nabla \times \mathbf{B}) \times \mathbf{B}=0 ; \\
\frac{\partial}{\partial t}\left(\frac{1}{2} \rho U^{2}+e+\frac{B^{2}}{2 \mu_{0}}\right)+\nabla \cdot\left[\left(\frac{1}{2} \rho U^{2}+\gamma e\right) \mathbf{U}\right. \\
\left.+\frac{1}{\mu_{0}} \mathbf{B} \times(\mathbf{U} \times \mathbf{B})\right]=0 ; \\
\quad \frac{\partial \mathbf{B}}{\partial t}=\nabla \times(\mathbf{U} \times \mathbf{B}) ; \quad \nabla \cdot \mathbf{B}=0,
\end{gathered}
$$

with an equation of state $e=\alpha n k_{B} T /(\gamma-1)$. The choice of $\alpha=2$ (or greater if incorporating the contribution of cosmic rays, etc.) corresponds to a plasma population comprising protons and electrons.

In setting $\mathbf{K}=0$ and $\left(\eta_{k l}\right)=0$, we have implicitly assumed that PUIs are completely coupled to the thermal plasma. With $\mathbf{K} \neq 0$, heat conduction reduces the effective coupling of energetic particles to the thermal plasma, and their contribution to the total pressure is not as large. This will have important consequences for numerical models of, e.g., the large-scale heliosphere since they incorporate PUIs into the MHD equations, without distinguishing PUIs from thermal plasma and therefore neglect heat conduction. Consequently, the total pressure is over-estimated.

\section{Conclusions}

Observations by Voyager 1 and 2 and the IBEX spacecraft indicate that plasma in the outer heliosphere (the superand subsonic solar wind) and the VLISM possesses characteristics of a multi-component plasma, being essentially a non-equilibrated distribution of background thermal protons and electrons and PUIs of various origins. Limitations of space prevent discussion of all the observational threads that lead to this conclusion, and we list and discuss above only a few. In the supersonic solar wind region of the outer heliosphere, the anomalous heating of the solar wind (Williams et al. 1995) has been interpreted in terms of the dissipation of PUI-driven turbulence that leads to the heating of the solar wind plasma (Zank et al. 1996; 2012; Matthaeus et al. 1996, 1999; Smith et al. 2001; Adhikari et al. 2015a). In the inner heliosheath and the VLISM, the observed plasma characteristics of the HTS (Zank et al. 1996; Richardson 2008; Richardson et al. 2008) and the ENA observations made by IBEX (Zank et al. 2010; Desai et al. 2012, 2014; Zirnstein et al. 2014) have been similarly interpreted in terms of a multi-component plasma distribution comprising various PUI populations. Estimates of the collisional frequency between thermal plasma components and PUIs in the outer supersonic solar wind $(>\sim 10$ AU), IHS, and VLISM show that equilibration cannot be achieved in these regions. Illustrated in Fig. 4 is a schematic of the solar wind-LISM interaction region with colors indicating regions that have to be described in terms of a multi-component plasma. The three colors for the different regions indicate that each region has a distinct multi-component plasma description reflecting the different origins of the PUI population for each. In the

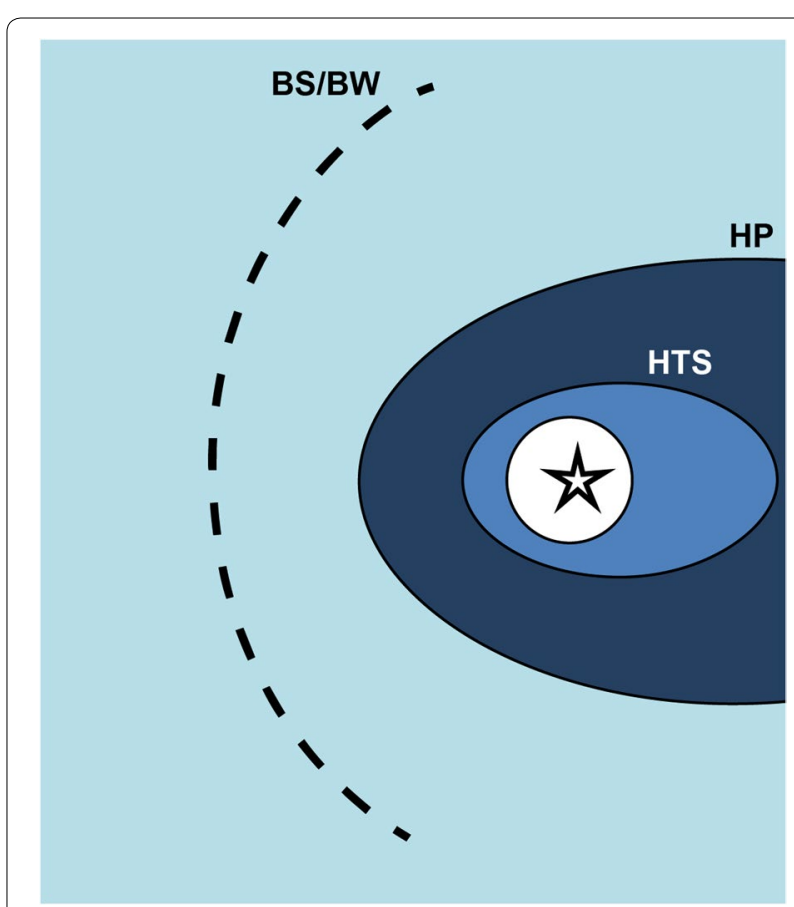

Fig. 4 Schematic of the solar wind-LISM interaction showing the boundaries. The colored regions require a non-equilibrated multicomponent plasma description. The different colors indicate that the non-equilibrated PUI component(s) originates from different physical processes. The region in white surrounding the Sun corresponds to the ionization cavity where PUls are not present in sufficient numbers to effectively mediate the plasma. See text and Table 1 for details 
supersonic solar wind, it is primarily PUIs created from interstellar neutral $\mathrm{H}$ that make up the PUI component. In the IHS, PUIs created in the supersonic solar wind and processed by the HTS are the most important PUI component energetically in the IHS, although there is a lower number density PUI component due to charge exchange with interstellar $\mathrm{H}$ as well. For the VLISM, the PUIs arise from charge exchange with secondary "solar wind or splash component neutrals" that were created in the supersonic solar wind and IHS. Basic plasma properties are mediated by PUIs in each of the regions illustrated in Fig. 4, and some discussion of the linear wave modes in these regions was presented by Zank et al. (2014). Table 1 provides a precise breakdown of the plasma models that are necessary for each region, together with estimates of the corresponding plasma and PUI temperature and density for each species (second column). Possible simplifications of the full model are listed in the third column for the IHS case.

Having motivated the need for a multi-component plasma description throughout the solar wind-VLISM interaction region, a derivation of the multi-fluid plasma model was presented, based on the analysis of Zank et al. (2014) (and correcting some typos). The standard approach of simply using a set of multi-fluid equations under the assumption that all distributions are isotropic, as done by Zieger et al. (2015), is incorrect in that it neglects the basic physics of PUI scattering by pre-existing and self-excited fluctuations. Numerous observations of the flat-topped form of the PUI distribution in the solar wind show that wave-particle scattering is fundamental to the physics of PUIs - for a review, see Zank (1999). Wave-particle scattering of PUIs introduces a collisionless form of PUI heat conduction and viscosity through the PUI pressure tensor. These important dissipative terms need to be included in any description of a non-equilibrated PUI-mediated plasma. The model presented here is appropriate for use in models of the global heliosphere. In particular, in the inner heliosheath, the role of PUI and ACR heat flux is to partially decouple the full pressure contribution of the PUIs and energetic particles from the overall pressure, thereby reducing the effective thermodynamic pressure balancing the interstellar pressure against that of the inner heliosheath. The net effect should be a thinner heliosheath than predicted by conventional MHD models that over-estimate the total pressure contribution contributed by energetic particles. The new model equations should be used to explore the global structure of the solar wind-VLISM interaction eventually (but this is well beyond the current scope of the paper). Finally, we note that it is not completely obvious how or whether ENA fluxes would change. At leading order, the energy densities of the different ionized components should be largely unchanged in the IHS. The only difference is that the heat flux associated with energetic particles removes energy (i.e., reduces their pressure contribution) from the overall total pressure of the inner heliosheath. Because this could lead to a thinner heliosheath, the ENA flux could be reduced but because the heat flux causes the energetic ionized particles to diffuse to greater distances; the net effect may be that there is little change in the overall ENA fluxes.

\section{Table 1 An explicit listing of the possible separate PUI populations for the different regions illustrated in Fig. 4}

\begin{tabular}{|c|c|c|}
\hline Region & Plasma model & Reduced model \\
\hline $\begin{array}{l}<8 \mathrm{AU} \text { (within ionization } \\
\text { cavity) }\end{array}$ & Standard MHD model_-PUIs essentially treated as test particles & \\
\hline $\begin{array}{l}\text { 8 AU to HTS (i.e., beyond } \\
\text { ionization cavity) }\end{array}$ & $\begin{array}{l}\text { Multi-component model } \\
\text { Interstellar PUls created in solar wind }\left(T \leq 1 \mathrm{keV}, n_{p} \sim 0.05-0.2 n_{i}\right)+\text { ther- } \\
\text { mal plasma (protons and electrons) }\end{array}$ & \\
\hline IHS & $\begin{array}{l}\text { Multi-component model } \\
\text { Three PUI populations: } \\
\text { 1) Interstellar PUIs transmitted across HTS without reflection }(T \sim 1 \mathrm{keV} \text {, } \\
\left.n_{p} \sim 0.18 n_{i}\right) \\
\text { 2) Interstellar PUIs reflected and then transmitted at HTS }(T \sim 6-7 \mathrm{keV} \text {, } \\
\left.n_{p} \sim 0.02 n_{i}\right) \\
\text { 3) Interstellar PUIs created in IHS }\left(T \sim 50 \mathrm{eV}, n_{p} \sim 0.015 n_{i}\right)+\text { thermal } \\
\text { plasma }\left(T \sim 16 \mathrm{eV}, n_{i} \sim 0.005 \mathrm{~cm}^{-3}\right)\end{array}$ & $\begin{array}{l}\text { Multi-component model } \\
\text { 1) Retain 1) + 2) and incorporate } 3 \text { ) in } \\
\text { thermal plasma model (i.e., neglect heat } \\
\text { flux for } T \sim 50 \mathrm{eV} \text { PUls) } \\
\text { 2) Combine models 1) and 2) and incorpo- } \\
\text { rate 3) in thermal plasma model }\end{array}$ \\
\hline VLISM & $\begin{array}{l}\text { Multi-component model } \\
\text { Solar wind PUls created in VLISM }\left(T \sim 200 \mathrm{eV}, n_{p} \sim 5 \times 10^{-5} \mathrm{~cm}^{-3}\right)+ \\
\text { thermal plasma }\left(T \sim 0.65 \mathrm{eV}, n_{i} \sim 0.15 \mathrm{~cm}^{-3}\right)\end{array}$ & \\
\hline
\end{tabular}

Characteristic temperatures and densities are given for the different PUI species. In estimating $n_{p}$ for interstellar PUls created in the IHS, we assumed a characteristic time scale of $40 \mathrm{AU} / 100 \mathrm{~km} / \mathrm{s} \sim 6 \times 10^{7} \mathrm{~s}$ 


\section{Acknowledgements}

We acknowledge the partial support of NASA Grants NNX08AJ33G, Subaward 37102-2, NNX14AC08G, NNX14AJ53G, A99132BT, RR185-447/4944336 and NNX12AB30G.

\section{Competing interests}

The author declares that he has no competing interests.

Received: 15 January 2016 Accepted: 23 June 2016 Published online: 15 July 2016

\section{References}

Adhikari L, Zank GP, Bruno R, Telloni D, Hunana P, Dosch A, Marino R, Hu $Q$ (2015) The transport of low-frequency turbulence in astrophysical flows. II. Solutions for the super-alfvénic solar wind. Astrophys J 805:63. doi:10.1088/0004-637X/805/1/63

Axford WI, Leer E, McKenzie JF (1982) The structure of cosmic ray shocks. Astron Astrophys 111:317-325

Burlaga LF, Ness NF, Belcher JW, Szabo A, Isenberg PA, Lee MA (1994) Pickup protons and pressure-balanced structures: vyager 2 observations in merged in teractionregionsnear $35 \mathrm{AU}$. J Geophys Res 99:21511. doi:10.1029/94JA01999

Burlaga LF, Ness NF, Acuña MH, Lepping RP, Connerney JEP, Richardson D (2008) Magnetic fields at the solar wind termination shock. Nature 454:75-77. doi:10.1038/nature07029

Burlaga LF, Ness NF, Gurnett DA, Kurth WS (2013) Evidence for a shock in interstellar plasma: voyager 1. Astrophys J Lett 778:3. doi:10.1088/2041-8205/778/1/L3

Burrows RH, Zank GP, Webb GM, Burlaga LF, Ness NF (2010) Pickup ion dynamics at the heliospheric termination shock observed by voyager 2 . Astrophys J 715:1109-1116. doi:10.1088/0004-637X/715/2/1109

Bzowski M, Swaczyna P, Kubiak MA, Sokół JM, Fuselier SA, Galli A, Heirtzler D, Kucharek H, Leonard TW, McComas DJ, Möbius E, Schwadron NA, Wurz P (2015) Interstellar neutral helium in the heliosphere from IBEX observations. III. Mach number of the flow, velocity vector, and temperature from the first six years of measurements. Astrophys J Suppl Ser 220:28. doi:10.1088/0067-0049/220/2/28.arxiv:1510.04835

Cairns IH, Zank P (2002) Turn-on of 2-3kHz radiation beyond the heliopause. Geophys Res Lett 29:47. doi:10.1029/2001GL014112

Cannon BE, Smith CW, Isenberg PA, Vasquez BJ, Murphy N, Nuno RG (2014) Ulysses observations of magnetic waves due to newborn interstellar pickup ions. I. New observations and linear analysis. Astrophys J 784:150. doi:10.1088/0004-637X/784/2/150

Decker RB, Krimigis SM, Roelof EC, Hill ME (2015) Recent particle measurements from voyagers 1 and 2. J Phys Conf Ser 577(1):012006. doi:10.1088/1742-6596/577/1/012006

Desai MI, Allegrini FA, Dayeh MA, DeMajistre B, Funsten H, Heerikhuisen J, McComas DJ, Pogorelov N, Schwadron NA, Zank GP (2012) Spectral properties of 0.5-6 kev energetic neutral atoms measured by the interstellar boundary explorer (IBEX) along the lines of sight of voyager. Astrophys $J$ Lett 749:30. doi:10.1088/2041-8205/749/2/L30

Desai MI, Allegrini FA, Bzowski M, Dayeh MA, Funsten H, Fuselier SA, Heerikhuisen J, Kubiak MA, McComas DJ, Pogorelov NV, Schwadron NA, Sokół JM, Zank GP, Zirnstein EJ (2014) Energetic neutral atoms measured by the interstellar boundary explorer (IBEX): evidence for multiple heliosheath populations. Astrophys J 780:98. doi:10.1088/0004-637X/780/1/98

Fisk LA, Gloeckler G (2009) The acceleration of anomalous cosmic rays by stochastic acceleration in the heliosheath. Adv Space Res 43:1471-1478. doi:10.1016/j.asr.2009.02.010

Fuselier SA, Allegrini F, Bzowski M, Funsten HO, Ghielmetti AG, Gloeckler G, Heirtzler D, Janzen P, Kubiak M, Kucharek H, McComas DJ, Möbius E, Moore TE, Petrinec SM, Quinn M, Reisenfeld D, Saul LA, Scheer JA, Schwadron N, Trattner KJ, Vanderspek R, Wurz P (2012) Heliospheric neutral atom spectra between 0.01 and $6 \mathrm{keV}$ from IBEX. Astrophys J 754:14. doi:10.1088/0004-637X/754/1/14

Gary P (1991) Electromagnetic ion/ion instabilities and their consequences in space plasmas - a review. Space Sci Rev 56:373-415. doi:10.1007/ BF00196632
Gary SP, Madland CD (1988) Electromagnetic ion instabilities in a cometary environment. J Geophys Res 93:235-241. doi:10.1029/JA093iA01p00235

Gloeckler G, Geiss J, Roelof EC, Fisk LA, I pavich FM, Ogilvie KW, Lanzerotti LJ, vonSteiger R, Wilken B (1994) Acceleration of interstellar pickup ions in the disturbed solarwind observed on ULYSSES. J Geophys Res 99:17. doi:10.1029/94JA01509

Gloeckler G, Geiss J, Fisk LA (2001) Heliospheric and interstellar phenomena revealed from observations of pickup ions. In: Balogh A, Marsden RG, Smith EJ (eds) The heliosphere near solar minimum. The Ulysses perspective. Springer-Praxis books in astrophysics and astronomy. Springer, London, pp 287-326

Gurnett DA, Kurth WS, Burlaga LF, Ness F (2013) Science 2025:150-153. doi:10.1126/science.1241681

Heerikhuisen J, Pogorelov NV, Florinski V, Zank GP, leRoux JA (2008) The effects of a $\kappa$-distribution in the heliosheath on the global heliosphere and ENA flux at 1 AU. Astrophys J 682:679-689. doi:10.1086/588248. arxiv:0803.2538

Heerikhuisen J, Zirnstein EJ, Funsten HO, Pogorelov NV, Zank GP (2014) The effect of new interstellar medium parameters on the heliosphere and energetic neutral atoms from the interstellar boundary. Astrophys J 784:73. doi:10.1088/0004-637X/784/1/73

Holzer TE (1972) Interaction of the solar wind with the neutral component of the interstellar gas. J Geophys Res 77:5407. doi:10.1029/JA077i028p05407

Holzer TE (1989) Interaction between the solar wind and the interstellar medium. Ann Rev Astron Astrophys 27:199-234. doi:10.1146/annurev aa.27.090189.001215

Isenberg PA (1986) Interaction of the solar wind with interstellar neutral hydrogen — three-fluid model. J Geophys Res 91:9965-9972. doi:10.1029/ JA091iA09p09965

Isenberg PA (1995) Interstellar pick u pions: not just theory anymore. Rev Geophys 33:623-627. doi:10.1029/95RG00553

Isenberg PA (1997) A hemispherical model of an isotropic interstellar pickup ions. J Geophys Res 102:4719-4724. doi:10.1029/96JA03671

Krimigis SM, Mitchell DG, Roelof EC, Hsieh KC, McComas DJ (2009) Imaging the interaction of the heliosphere with the interstellar medium from Saturn with Cassini. Science 326:971. doi:10.1126/science.1181079

Krimigis SM, Decker RB, Roelof EC, Hill ME, Armstrong TP, Gloeckler G, Hamilton DC, Lanzerotti LJ (2013) Search for the exit: voyager 1 at heliosphere's border with the galaxy. Science 341:144-147. doi:10.1126/ science.1235721

Lazarian A, Opher M (2009) A model of acceleration of anomalous cosmic rays by reconnection in the heliosheath. Astrophys J 703:8-21. doi:10.1088/0004-637X/703/1/8.arxiv:0905.1120

Lee MA, Ip WH (1987) Hydromagnetic wave excitation by ionised interstellar hydrogen and helium in the solar wind. J Geophys Res 92:11041-11052. doi:10.1029/JA092iA10p11041

Lipatov AS, Zank GP (1999) Pickup Ion acceleration at low- $\beta_{p}$ perpendicular shocks. Phys Rev Lett 82:3609-3612. doi:10.1103/PhysRevLett.82.3609

Matsukiyo S, Scholer M (2011) Microstructure of the heliospheric termination shock: full particle electrodynamic simulations. J Geophys Res 116:08106. doi:10.1029/2011JA016563

Matsukiyo S, Scholer M (2014) Simulations of pickup ion mediated quasi-perpendicular shocks: implications for the heliospheric termination shock. J Geophys Res 119:2388-2399. doi:10.1002/2013JA019654

Matthaeus WH, Zank GP, Oughton S (1996) Phenomenology of hydromagnetic turbulence in a uniformly expanding medium. J Plasma Phys 56:659-675. doi:10.1017/S0022377800019516

Matthaeus WH, Zank GP, Smith CW, Oughton S (1999) Turbulence, spatial transport, and heating of the solar wind. Phys Rev Lett 82:3444-3447. doi:10.1103/PhysRevLett.82.3444

McComas DJ, Funsten HO, Fuselier SA, Lewis WS, Möbius E, Schwadron A (2011) IBEX observations of heliospheric energetic neutral atoms: current understanding and future directions. Geophys Res Lett 38:18101. doi:10.1 029/2011GL048763

McComas DJ, Alexashov D, Bzowski M, Fahr H, Heerikhuisen J, Izmodenov V, Lee MA, Möbius E, Pogorelov N, Schwadron NA, Zank GP (2012) The heliosphere's interstellar interaction: no bow shock. Science 336:1291. doi:10.1126/science.1221054

McComas DJ, Bzowski M, Fuselier SA, Frisch PC, Galli A, Izmodenov W, Katushkina OA, Kubiak MA, Lee MA, Leonard TW, Möbius E, Park J, Schwadron NA, Sokół JM, Swaczyna P, Wood BE, Wurz P (2015) Local interstellar 
medium: six years of direct sampling by IBEX. Astrophys I Suppl Ser 220:22. doi:10.1088/0067-0049/220/2/22

Oka M, Zank GP, Burrows RH, Shinohara I (2011) Energy dissipation at the termination shock: 1DPIC simulation. In: Florinski V, Heerikhuisen J, Zank GP, Gallagher DL (eds)American Institute of Physics Conference Series, vol. 1366, p 53-59. doi:10.1063/1.3625589

RB Decker, Krimigis SM, Roelof EC, Hill ME, Armstrong TP, Gloeckler G, Hamilton DC, Lanzerotti $\sqcup J$ (2008) Mediation of the solar wind termination shock by non-thermal ions. Nature 454:67-70. doi:10.1038/nature07030

Richardson JD (2009) Heliospheric shocks and sheaths. In: Ao X, Burrows GZR (eds) American Institute of Physics ConferenceSeries, vol. 1183, p 147-155. doi:10.1063/1.3266771

Richardson JD, Paularena KI, Lazarus AJ, Belcher JW (1995) Evidence for a solar wind slowdown in the outer heliosphere? Geophys Res Lett 22:14691472. doi:10.1029/95GL01421

Richardson JD (2008) Plasma temperature distributions in the heliosheath. Geophys Res Lett 35:23104. doi:10.1029/2008GL036168

Richardson JD, Kasper JC, Wang C, Belcher JW, Lazarus AJ (2008) Cool heliosheath plasma and deceleration of the upstream solar wind at the termination shock. Nature 454:63-66. doi:10.1038/nature07024

Schwadron NA, Möbius E, Leonard T, Fuselier SA, McComas DJ, Heirtzler D, Kucharek H, Rahmanifard F, Bzowski M, Kubiak MA, Sokół JM, Swaczyna P, Frisch P (2015) Determination of interstellar He parameters using five years of data from the IBEX: beyond closed form approximations. Astrophys J Suppl Ser 220:25. doi:10.1088/0067-0049/220/2/25

Senanayake UK, Florinski V, Cummings AC, Stone EC (2015) Spectral evolution of anomalous cosmic rays at voyager 1 beyond the termination shock. Astrophys J 12:804. doi:10.1088/0004-637X/804/1/12

Smith CW, Matthaeus WH, Zank GP, Ness NF, Oughton S, Richardson JD (2001) Heating of the low-latitude solar wind by dissipation of turbulent magnetic fluctuations. J Geophys Res 106:8253-8272. doi:10.1029/200 OJA000366

Stone EC, Cummings AC, McDonald FB, Heikkila BC, Lal N, Webber R (2013) Voyager 1 observes low-energy galactic cosmic rays in a region depleted of heliospheric ions. Science 341:150-153. doi:10.1126/science.1236408

Webb M (1983) The structure of oblique MHD cosmic-ray shocks. Astron Astrophys 127:97-112

Williams LL, Zank GP, Matthaeus WH (1995) Dissipation of pickup-induced waves: a solar wind temperature increase in the outer heliosphere? J Geophys Res 100:17059-17068. doi:10.1029/95JA01261

Williams LL, Zank GP (1994) Effect of magnetic field geometry on the wave signature of the pickup of interstellar neutrals. J Geophys Res 99:19229. doi:10.1029/94JA01657

Wu P, Winske D, Gary SP, Schwadron NA, Lee MA (2009) Energy dissipation and ion heating at the heliospheric termination shock. J Geophys Res 114:08103. doi:10.1029/2009JA014240

Yang Z, Liu YD, Richardson JD, Lu Q, Huang C, Wang R (2015) Impact of pickup ions on the shock front nonstationarity and energy dissipation of the heliospheric termination shock: two-dimensional full particle simulations and comparison with voyager 2 observations. Astrophys J 809:28. doi:10.1088/0004-637X/809/1/28.arxiv:1506.08492
Zank GP, Pauls HL, Cairns IH, Webb GM (1996) Interstellar pickup ions and quasi-perpendicular shocks: implications for the termination shock and interplanetary shocks. J Geophys Res 101:457-478. doi:10.1029/95JA02860

Zank GP, Pauls HL, Williams LL, Hall DT (1996) Interaction of the solar wind with the local interstellar medium: a multifluid approach. J Geophys Res 101:21639-21656. doi:10.1029/96JA02127

Zank GP, Matthaeus WH, Smith CW (1996) Evolution of turbulent magnetic fluctuation power with heliospheric distance. J Geophys Res 101:1709317108. doi:10.1029/96JA01275

Zank GP (1999) Interaction of the solar wind with the local interstellar medium: a theoretical perspective. Space Sci Rev 89:413-688. doi:10.102 3/A:1005155601277

Zank GP, Pogorelov NV, Heerikhuisen J, Washimi H, Florinski V, Borovikov S, Kryukov I, Müller HR (2009) Physics of the solar wind-local interstellar medium interaction: role of magnetic fields. Space Sci Rev 146:295-327. doi:10.1007/s11214-009-9497-6

Zank GP, Heerikhuisen J, Pogorelov NV, Burrows R, McComas D (2012) Microstructure of the heliospheric termination shock: implications for energetic neutral atom observations. Astrophys J 708:1092-1106. doi:10.1088/0004-637X/708/2/1092

Zank GP, Dosch A, Hunana P, Florinski V, Matthaeus WH, Webb GM (2012) The transport of low-frequency turbulence in astrophysical flows. I. Governing equations. Astrophys J 35:745. doi:10.1088/0004-637X/745/1/35

Zank GP, Heerikhuisen J, Wood BE, Pogorelov NV, Zirnstein E, McComas DJ (2013) Heliospheric structure: the bow wave and the hydrogen wall. Astrophys J 763:20. doi:10.1088/0004-637X/763/1/20

Zank GP, Hunana P, Mostafavi P, Goldstein ML (2014) Pickup ion mediated plasmas. I. basic model and linear waves in the solar wind and local interstellar medium. Astrophys J 797:87. doi:10.1088/0004-637X/797/2/87

Zank GP (2014) Transport processes in space physics and astrophysics. Lecture notes in physics, vol 877. Springer, Berlin. doi:10.1007/978-1-4614-8480-6

Zank GP, Hunana P, Mostafavi P, Le Roux JA, Li G, Webb GM, Khabarova O, Cummings A, Stone E, Decker R (2015) Diffusive shock acceleration and reconnection acceleration processes. Astrophys J 814:137. doi:10.1088/0004-637X/814/2/137

Zank P (2015) Faltering steps into the galaxy: the boundary regions of the heliosphere. Ann Rev Astron Astrophys 53:449-500. doi:10.1146/ annurev-astro-082214-122254

Zieger B, Opher M, Tóth G, Decker RB, Richardson JD (2015) Constraining the pickup ion abundance and temperature through the multifluid reconstruction of the Voyager 2 termination shock crossing. J Geophys Res 120:7130-7153. doi:10.1002/2015JA021437

Zirnstein EJ, Heerikhuisen J, Zank GP, Pogorelov NV, McComas DJ, Desai MI (2014) Charge-exchange coupling between pickup ions across the heliopause and its effect on energetic neutral hydrogen flux. Astrophys J 783:129. doi:10.1088/0004-637X/783/2/129

\section{Submit your manuscript to a SpringerOpen ${ }^{\circ}$ journal and benefit from:}

- Convenient online submission

- Rigorous peer review

- Immediate publication on acceptance

- Open access: articles freely available online

- High visibility within the field

- Retaining the copyright to your article

Submit your next manuscript at $\boldsymbol{\nabla}$ springeropen.com 